\title{
Integral Representation for Borel Sum of Divergent Solution to a Certain Non-Kowalevski Type Equation
}

By

\author{
Kunio ICHINOBE*
}

\begin{abstract}
We shall develop the theory of Borel summability or $k$-summability for a divergent solution of the Cauchy problem for non-Kowalevskian equations of quasihomogeneous type. Precisely, we first establish necessary and sufficient conditions for the Borel summability in terms of the Cauchy data (cf. Theorem 2.1), and next we give an integral representation of the Borel sum by using kernel functions which are given by Meijer $G$-function or the generalized hypergeometric functions of confluent type (cf. Theorems 2.3 and 2.6).
\end{abstract}

\section{$\S 1 . \quad$ Introduction}

Throughout this paper, we denote $(t, x) \in \mathbb{C}^{2}$ and $\left(\partial_{t}, \partial_{x}\right)=(\partial / \partial t, \partial / \partial x)$. We consider the following Cauchy problem for a partial differential equation of non-Kowalevski type

$$
\left\{\begin{array}{l}
Q\left(\partial_{t}, \partial_{x}\right) U(t, x) \equiv\left(\partial_{t}^{d}-\sum_{j=1}^{d} a_{j} \partial_{t}^{d-j} \partial_{x}^{r j}\right) U(t, x)=0, \\
\partial_{t}^{k} U(0, x)=0(k=0,1, \ldots, d-2), \quad \partial_{t}^{d-1} U(0, x)=\varphi(x),
\end{array}\right.
$$

where $d(\geq 1) \in \mathbb{N}=\{1,2,3, \ldots\}, a_{j} \in \mathbb{C}(j=1,2, \ldots, d), r(>1) \in \mathbb{Q}$ and the Cauchy data $\varphi(x)$ is holomorphic in a neighbourhood of the origin. Here we assume $a_{j}=0$ if $r j \in \mathbb{Q} \backslash \mathbb{N}$.

Communicated by T. Kawai. Received April 8, 2002. Revised September 12, 2002 and October 18, 2002.

2000 Mathematics Subject Classification(s): primary 35C15, secondary 35E15, 35C20.

*Graduate School of Mathematics, Nagoya University, Nagoya, 464-8602, Japan.

e-mail: m95006@math.nagoya-u.ac.jp 
Our purpose in this paper is to investigate necessary and sufficient conditions for the Borel summability of a formal power series solution with respect to $t$-variable of the Cauchy problem (1.1), which is divergent in general (Subsection 2.5). The main interest of this paper is to give an integral representation of the Borel sum of the formal power series solution under the conditions.

The motivation of our study comes from the following background: The theory of Borel summability or $k$-summability or multisummability for divergent power series solutions of ordinary differential equations with irregular singular points has been developed extensively from the 1980's and the development of the theory can be found in the book [Bal 1].

For partial differential equations, the study of formal power series solutions has been developed by many authors for various type equations ([Miy 1], [Miy 2], [GT], [Shi] and references therein). Ōuchi [Ōuc] studied the existence of asymptotic solutions of divergent solution for general equations, and showed that infinitely many solutions exist in a sectorial domain of small opening angle in the plane of one variable. If we ask the existence of Gevrey asymptotic solutions in a sector of large opening angle, we can not expect the existence of such solutions in general. But it is unique if it does exist (cf. the definition of the Borel summability, Subsection 2.7). From such a point of view, Lutz-Miyake-Schäfke [LMS] studied the formal solution of the Cauchy problem of the complex heat equation, and gave the characterization of the Borel summability of the formal power series solution in $t$ variable in terms of the global conditions for the Cauchy data. After that, their result was generalized by Balser [Bal 2] under more general conditions upon the Cauchy data, and Balser-Miyake $[\mathrm{BM}]$ proved a sufficient condition for the Borel summability of divergent power series in $t$ variable. Their result includes the Cauchy problem of partial differential equations of non-Kowalevski type. In [Miy 3], Miyake proved that the sufficient condition is actually a necessary condition for some class of equations. Under the conditions for the Cauchy data given in [BM] and [Miy 3] the explicit integral representation of the Borel sum of the formal solution was given by Ichinobe [Ich].

In this paper we shall extend the results in [Ich]. We shall give the characterization of the Borel summability for the formal solution of the Cauchy problem for a quasi-homogeneous partial differential operator (see Theorem 2.1), and give an explicit integral representation of its Borel sum (see Theorems 2.3 and 2.6) under the same conditions upon the Cauchy data. 


\section{$\S 2$. Statement of Results}

\section{$\S 2.1$. Reduction of the equation}

Let $r=q / p,(q>p \geq 1)$ be the irreducible fraction, and put

$$
d=p \nu+\nu_{0} \quad\left(0 \leq \nu_{0}<p\right) .
$$

Then the given differential operator $Q\left(\partial_{t}, \partial_{x}\right)$ is written in the following form

$$
\begin{aligned}
Q\left(\partial_{t}, \partial_{x}\right) & =\partial_{t}^{d}-a_{p} \partial_{t}^{d-p} \partial_{x}^{q}-a_{2 p} \partial_{t}^{d-2 p} \partial_{x}^{2 q}-\cdots-a_{\nu p} \partial_{t}^{d-\nu p} \partial_{x}^{\nu q} \\
& =\left(\partial_{t}^{p \nu}-\sum_{j=1}^{\nu} a_{j p} \partial_{t}^{p(\nu-j)} \partial_{x}^{j q}\right) \partial_{t}^{\nu_{0}} \equiv P\left(\partial_{t}, \partial_{x}\right) \partial_{t}^{\nu_{0}}
\end{aligned}
$$

Therefore by replacing $\partial_{t}^{\nu_{0}} U(t, x)$ and $a_{j p}$ by $u(t, x)$ and $a_{j}$, respectively, the Cauchy problem (1.1) is reduced to the following one.

$$
\left\{\begin{array}{l}
P\left(\partial_{t}, \partial_{x}\right) u(t, x) \equiv\left(\partial_{t}^{p \nu}-\sum_{j=1}^{\nu} a_{j} \partial_{t}^{p(\nu-j)} \partial_{x}^{j q}\right) u(t, x)=0, \\
\partial_{t}^{k} u(0, x)=0(k=0,1, \ldots, p \nu-2), \quad \partial_{t}^{p \nu-1} u(0, x)=\varphi(x) .
\end{array}\right.
$$

\section{§2.2. Characteristic equation}

For the operator $P$, let us consider the following algebraic equation, which is called a characteristic equation

$$
\sigma^{\nu}-\sum_{j=1}^{\nu} a_{j} \sigma^{\nu-j}=0
$$

Let $\left\{\alpha_{j}\right\}_{j=1}^{\mu}\left(\alpha_{j} \neq 0\right)$ be the set of the distinct root $\alpha_{j}$ (characteristic root) of $\ell_{j}$-multiplicity with $\sum_{j=1}^{\mu} \ell_{j}=\nu$. Then the operator $P$ is rewritten by

$$
P \equiv \partial_{t}^{p \nu}-\sum_{j=1}^{\nu} a_{j} \partial_{t}^{p(\nu-j)} \partial_{x}^{j q}=\prod_{j=1}^{\mu} P_{j}^{\ell_{j}}, \quad P_{j}=P_{j}\left(\partial_{t}, \partial_{x}\right) \equiv \partial_{t}^{p}-\alpha_{j} \partial_{x}^{q} .
$$




\section{$\S 2.3$. Formal solution}

The Cauchy problem (2.3) has a unique formal solution $\hat{u}(t, x)$ which is given by

$$
\hat{u}(t, x)=\sum_{n \geq p \nu-1} u_{n}(x) \frac{t^{n}}{n !}=\sum_{n \geq 0} u_{p n+p \nu-1}(x) \frac{t^{p n+p \nu-1}}{(p n+p \nu-1) !} .
$$

If we put

$$
u_{p n+p \nu-1}(x)=A(n) \varphi^{(q n)}(x), \quad n \geq 0,
$$

then $A(n)$ satisfy the following difference equation

$$
A(n+\nu)-\sum_{j=1}^{\nu} a_{j} A(n+\nu-j)=0, \quad n=-\nu+1,-\nu+2, \ldots,
$$

with the Cauchy data

$$
A(-\nu+1)=A(-\nu+2)=\cdots=A(-1)=0, \quad A(0)=1 .
$$

Since a fundamental system of solutions of this difference equation is given by $\left\{n^{k-1} \alpha_{j}^{n} ; k=1,2, \ldots, \ell_{j}, j=1,2, \ldots, \mu\right\}, A(n)$ are given by

$$
A(n)=\sum_{j=1}^{\mu} \alpha_{j}^{n} \sum_{k=1}^{\ell_{j}} c_{j k} n^{k-1},
$$

with the coefficients $c_{j k}\left(j=1,2, \ldots, \mu, k=1,2, \ldots, \ell_{j}\right)$ satisfying the following linear system of equations

$$
\mathcal{A} \vec{c}=\vec{e},
$$

where $\mathcal{A}$ denotes a $\nu \times \nu$ matrix which is given by

$$
\begin{aligned}
& \mathcal{A}=
\end{aligned}
$$

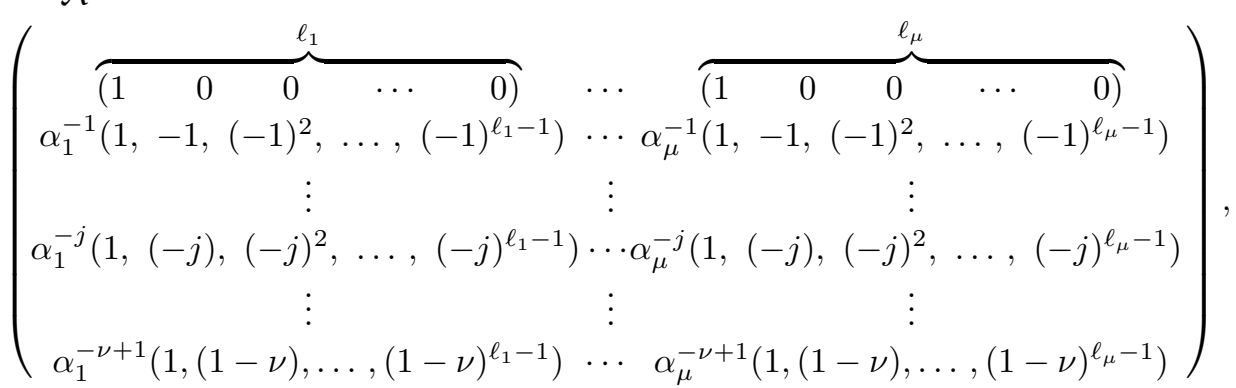


$\vec{c}={ }^{t}\left(c_{11}, c_{12}, \ldots, c_{1 \ell_{1}}, c_{21}, c_{22}, \ldots, c_{2 \ell_{2}}, \ldots, c_{\mu 1}, c_{\mu 2}, \ldots, c_{\mu \ell_{\mu}}\right)$ and $\vec{e}=$ ${ }^{t}(1,0,0, \ldots, 0)$.

Before stating our results, we shall prepare some definitions and notations.

\section{§2.4. Sector}

For $d \in \mathbb{R}, \beta>0$ and $\rho(0<\rho \leq \infty)$, we define a sector $S=S(d, \beta, \rho)$ by

$$
S(d, \beta, \rho):=\left\{t \in \mathbb{C} ;|d-\arg t|<\frac{\beta}{2}, 0<|t|<\rho\right\},
$$

where $d, \beta$ and $\rho$ are called the direction, the opening angle and the radius of $S$, respectively.

\section{$\S 2.5$. Gevrey formal power series}

We denote by $\mathcal{O}[[t]]$ the ring of formal power series in $t$-variable with coefficients in $\mathcal{O}$ (the set of holomorphic functions at $x=0$ ).

For $k>0$, we define $\mathcal{O}[[t]]_{1 / k}$, the ring of formal power series of Gevrey order $1 / k$ in $t$-variable, in the following way: $\hat{f}(t, x)=\sum_{n=0}^{\infty} f_{n}(x) t^{n} \in \mathcal{O}[[t]]_{1 / k}$ $(\subset \mathcal{O}[[t]])$ if and only if the coefficients $f_{n}(x)$ are holomorphic on a common closed disk $B_{r}:=\{x \in \mathbb{C} ;|x| \leq r\}$ and there exist some positive constants $C$ and $K$ such that for any $n$, we have

$$
\max _{|x| \leq r}\left|f_{n}(x)\right| \leq C K^{n} \Gamma\left(1+\frac{n}{k}\right),
$$

where $\Gamma$ denotes the Gamma function which is defined by the following Gamma integral

$$
\Gamma(z)=\int_{0}^{\infty} t^{z-1} e^{-t} d t, \quad \operatorname{Re} z>0 .
$$

By using this terminology, we can easily prove that our formal solution $\hat{u}(t, x)$ of the Cauchy problem (2.3) belongs to $\mathcal{O}[[t]]_{(q-p) / p}$, that is, $k=p /(q-p)$.

\section{§2.6. Gevrey asymptotic expansion}

Let $k>0, \hat{f}(t, x)=\sum_{n=0}^{\infty} f_{n}(x) t^{n} \in \mathcal{O}[[t]]_{1 / k}$ and $f(t, x)$ be an analytic function on $S(d, \beta, \rho) \times B_{r}$. Then we define that

$$
f(t, x) \cong_{k} \hat{f}(t, x) \text { in } S=S(d, \beta, \rho),
$$


if for any closed subsector $S^{\prime}$ of $S$, there exist some positive constants $C$ and $K$ such that for any $N$, we have

$$
\max _{|x| \leq r}\left|f(t, x)-\sum_{n=0}^{N-1} f_{n}(x) t^{n}\right| \leq C K^{N}|t|^{N} \Gamma\left(1+\frac{N}{k}\right), \quad t \in S^{\prime} .
$$

\section{$\S 2.7$. Borel summability}

For $k>0, d \in \mathbb{R}$ and $\hat{f}(t, x) \in \mathcal{O}[[t]]_{1 / k}$, we define that $\hat{f}(t, x)$ is $k$ summable or Borel summable in $d$ direction if there exist a sector $S=S(d, \beta, \rho)$ with $\beta>\pi / k$ and an analytic function $f(t, x)$ on $S \times B_{r}$ such that $f(t, x) \cong_{k}$ $\hat{f}(t, x)$ in $S$.

We remark that such a function $f(t, x)$ is unique, if it exists, for a $k$ summable series $\hat{f}(t, x)$. Therefore the function $f(t, x)$ is called the Borel sum of $\hat{f}(t, x)$ in $d$ direction and it is written by $f^{d}(t, x)$. For the detail of the theory of Borel summability or $k$-summability, see the book [Bal 1, p. 24].

\section{$\S 2.8$. Characterization of Borel summability}

Let $\left\{\alpha_{j}\right\}_{j=1}^{\mu} \subset \mathbb{C} \backslash\{0\}$ be the roots of the characteristic equation (2.4). For a direction $d \in \mathbb{R}$ and an opening angle $\varepsilon$, we define multi-sectors $\Omega_{\alpha_{j}}$ and $\Omega_{x}$ by

$$
\begin{gathered}
\Omega_{\alpha_{j}}=\Omega_{\alpha_{j}}(p, q ; d, \varepsilon):=\bigcup_{m=0}^{q-1} S\left(\frac{p d+\arg \alpha_{j}+2 \pi m}{q}, \varepsilon, \infty\right), \\
j=1,2, \ldots, \mu,
\end{gathered}
$$

$$
\Omega_{x}=\Omega_{x}(p, q, \mu ; d, \varepsilon):=\bigcup_{j=1}^{\mu} \Omega_{\alpha_{j}}(p, q ; d, \varepsilon) .
$$

We remark that $\Omega_{\alpha_{j}}$ is a union of $q$ sectors and $\Omega_{x}$ is a union of $q \mu$ sectors, and if $\arg \alpha_{j}=\arg \alpha_{k}$ for any $j$ and $k$, then we have $\Omega_{x}=$ $\Omega_{\alpha_{j}}$. 


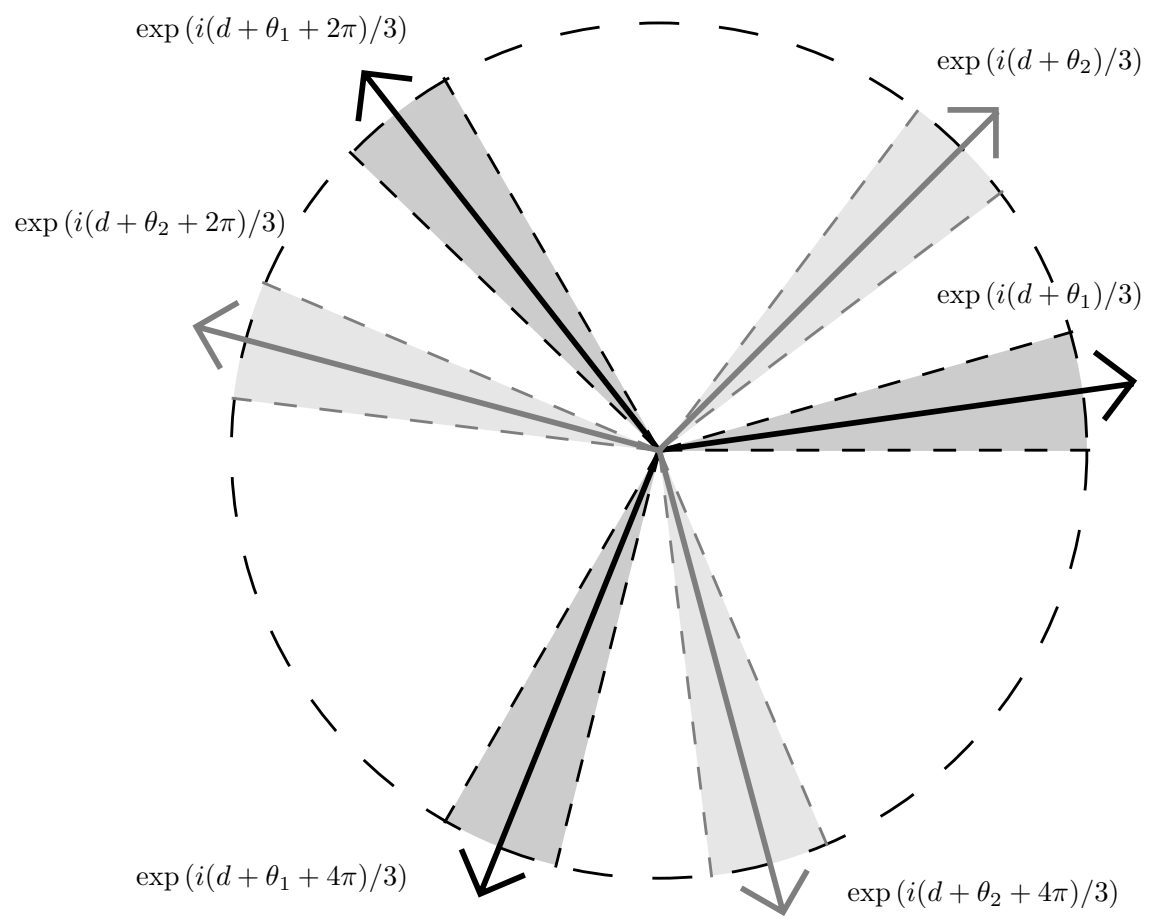

Figure 1. $\Omega_{x}=\Omega_{x}(1,3,2 ; d, \varepsilon) ; \theta_{j}=\arg \alpha_{j}(j=1,2)$

Now, our first result for the Borel summability is stated as follows.

Theorem 2.1 (Borel summability). Let $\hat{u}(t, x)$ be the formal solution of the Cauchy problem (2.3), which is given by (2.6). Then the following three conditions are equivalent:

(i) $\hat{u}(t, x)$ is $p /(q-p)$-summable in d direction.

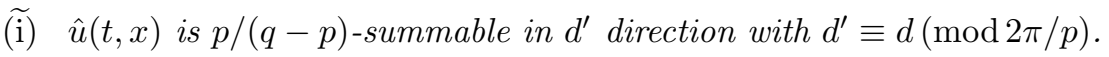

(ii) The Cauchy data $\varphi(x)$ can be continued analytically in $\Omega_{x}$ and has a growth condition of exponential order at most $q /(q-p)$ there, which means that there exist some positive constants $C$ and $\delta$ such that we have

$$
|\varphi(x)| \leq C \exp \left(\delta|x|^{q /(q-p)}\right), \quad x \in \Omega_{x}
$$

This result is a generalization of results in [LMS] and [Miy 3]. 
The equivalence between (i) and ( $\widetilde{\mathrm{i}})$ is trivial, since the operator $P\left(\partial_{t}, \partial_{x}\right)$ is invariant by the change of coordinate $t \rightarrow t e^{2 \pi i / p}$. Therefore the main task is to prove the equivalence between (i) and (ii), which will begin in Section 3 .

Remark 2.2. For the formal solution $\hat{U}(t, x)$ of the original Cauchy problem (1.1), Theorem 2.1 does hold, and the Borel sum $U^{d}(t, x)$ in $d$ direction of $\hat{U}(t, x)$ is given by $U^{d}(t, x)=\left(D_{t}^{-1}\right)^{\nu_{0}} u^{d}(t, x)$, that is, $\nu_{0}$ times iterated integral from 0 to $t$ of $u^{d}(t, x)$, where $u^{d}(t, x)$ denotes the Borel sum of $\hat{u}(t, x)$.

Our main purpose is to give an integral representation of the Borel sum $u^{d}(t, x)$ of the formal solution $\hat{u}(t, x)$ under the condition (ii) of Theorem 2.1 for the Cauchy data $\varphi(x)$.

Before stating our main results, we need some preparations for the special functions (cf. [Luk, p. 41, 144], [MS, p. 2]).

\section{§2.9. Generalized hypergeometric series}

For $\boldsymbol{\beta}=\left(\beta_{1}, \ldots, \beta_{p}\right) \in \mathbb{C}^{p}$ and $\boldsymbol{\gamma}=\left(\gamma_{1}, \ldots, \gamma_{q}\right) \in \mathbb{C}^{q}$, we define

$$
{ }_{p} F_{q}(\boldsymbol{\beta} ; \boldsymbol{\gamma} ; z)={ }_{p} F_{q}\left(\begin{array}{l}
\boldsymbol{\beta} \\
\boldsymbol{\gamma}
\end{array} ;\right):=\sum_{n=0}^{\infty} \frac{(\boldsymbol{\beta})_{n}}{(\boldsymbol{\gamma})_{n}} \frac{z^{n}}{n !},
$$

where

$$
(\boldsymbol{\beta})_{n}=\prod_{\ell=1}^{p}\left(\beta_{\ell}\right)_{n}, \quad(\gamma)_{n}=\prod_{j=1}^{q}\left(\gamma_{j}\right)_{n}, \quad(c)_{n}=\frac{\Gamma(c+n)}{\Gamma(c)}(c \in \mathbb{C}),
$$

(cf. [Luk, p. 41]).

We remark that ${ }_{p} F_{q}(\boldsymbol{\beta} ; \boldsymbol{\gamma} ; z)$ is a generalization of the Gauss hypergeometric series and satisfies the following ordinary differential equation

$$
E_{p, q}[\boldsymbol{\beta} ; \boldsymbol{\gamma}](f):=\left[\prod_{j=1}^{q} \delta_{z}\left(\delta_{z}+\gamma_{j}-1\right)-z \prod_{\ell=1}^{p}\left(\delta_{z}+\beta_{\ell}\right)\right] f(z)=0
$$

where $\delta_{z}=z(d / d z)$.

We suppose that $\beta_{\ell}, \gamma_{j} \notin \mathbb{Z}_{\leq 0}$ for all $\ell$ and $j$ where $\mathbb{Z}_{\leq 0}:=\{0,-1,-2, \ldots\}$. In each case where $p<q+1$ or $p=q+1$ or $p>q+1$, this formal power series represents an entire function or a convergent series in $|z|<1$ or a divergent series for all $z(\neq 0)$, respectively. 


\section{$\S 2.10$. Meijer $G$-function}

Let $0 \leq n \leq p, 0 \leq m \leq q$. For $\boldsymbol{\beta} \in \mathbb{C}^{p}$ and $\gamma \in \mathbb{C}^{q}$ with $\beta_{\ell}-\gamma_{j} \notin \mathbb{N}$ $(\ell=1,2, \ldots, n ; j=1,2, \ldots, m)$, we define

$$
G_{p, q}^{m, n}\left(z \mid \begin{array}{c}
\boldsymbol{\beta} \\
\gamma
\end{array}\right)=\frac{1}{2 \pi i} \int_{I} \frac{\prod_{j=1}^{m} \Gamma\left(\gamma_{j}+\tau\right) \prod_{\ell=1}^{n} \Gamma\left(1-\beta_{\ell}-\tau\right)}{\prod_{j=m+1}^{q} \Gamma\left(1-\gamma_{j}-\tau\right) \prod_{\ell=n+1}^{p} \Gamma\left(\beta_{\ell}+\tau\right)} z^{-\tau} d \tau,
$$

where $z^{-\tau}=\exp \{-\tau(\log |z|+i \arg z)\}$ and the path of integration $I$ runs from $\kappa-i \infty$ to $\kappa+i \infty$ for any fixed $\kappa \in \mathbb{R}$ in such a manner that, if $|\tau|$ is sufficiently large, then $\tau \in I$ lies on the line $\operatorname{Re} \tau=\kappa$, all poles of $\Gamma\left(\gamma_{j}+\tau\right)$, $\left\{-\gamma_{j}-k ; k \geq 0, j=1,2, \ldots, m\right\}$, lie to the left of the path and all poles of $\Gamma\left(1-\beta_{\ell}-\tau\right),\left\{1-\beta_{\ell}+k ; k \geq 0, \ell=1,2, \ldots, n\right\}$, lie to the right of the path. The integral converges absolutely on any compact set in the sector $S(0, \sigma \pi, \infty)$ if $\sigma=2(m+n)-(p+q)>0$. If $|\arg z|=\sigma \pi, \sigma \geq 0$, the integral converges absolutely when $p=q$ if $\operatorname{Re} \Delta<-1$; and when $p \neq q$, if with $\tau=\kappa+i \eta, \kappa$ and $\eta$ real, $\kappa$ is chosen so that for $\eta \rightarrow \pm \infty$,

$$
(p-q) \kappa>\operatorname{Re} \Delta+1+\frac{1}{2}(p-q)
$$

where

$$
\Delta=\sum_{j=1}^{q} \gamma_{j}-\sum_{\ell=1}^{p} \beta_{\ell}
$$

(cf. [MS, p.2], [Luk, p.144]).

We use the following abbreviations:

$$
\begin{gathered}
\boldsymbol{p}=(1,2, \ldots, p) \in \mathbb{N}^{p}, \quad \boldsymbol{q}=(1,2, \ldots, q) \in \mathbb{N}^{q} \\
\boldsymbol{p}+c=(1+c, 2+c, \ldots, p+c) \in \mathbb{C}^{p}, \quad c \in \mathbb{C} \\
\overline{\boldsymbol{p}}=\boldsymbol{p}-1=(0,1, \ldots, p-1) \\
\frac{\boldsymbol{p}}{p}=\left(\frac{1}{p}, \frac{2}{p}, \ldots, \frac{p}{p}\right) \\
\widehat{\boldsymbol{q}}_{\ell}=(1,2, \ldots, \ell-1, \ell+1, \ldots, q) \in \mathbb{N}^{q-1} \\
\boldsymbol{\Gamma}\left(\frac{\boldsymbol{p}}{p}\right)=\prod_{j=1}^{p} \Gamma\left(\frac{j}{p}\right) .
\end{gathered}
$$

In the following, the integration $\int_{0}^{\infty(\theta)}$ denotes the integration from 0 to $\infty$ along the half line of argument $\theta$. 


\section{$\S 2.11$. Integral representation of the Borel sum}

We recall that the formal solution $\hat{u}(t, x)$ is given by

$$
\begin{aligned}
\hat{u}(t, x) & =\sum_{j=1}^{\mu} \sum_{k=1}^{\ell_{j}} c_{j k} \sum_{n \geq 0} n^{k-1} \varphi^{(q n)}(x) \frac{\alpha_{j}^{n} t^{p n+p \nu-1}}{(p n+p \nu-1) !} \\
& =t^{p \nu-1} \sum_{j=1}^{\mu} \sum_{k=1}^{\ell_{j}} c_{j k} p^{1-k}\left(t \partial_{t}\right)^{k-1} \sum_{n \geq 0} \varphi^{(q n)}(x) \frac{\alpha_{j}^{n} t^{p n}}{(p n+p \nu-1) !} .
\end{aligned}
$$

Now, our main result is stated as follows.

Theorem 2.3 (Borel sum). Let $\left\{\alpha_{j}\right\}_{j=1}^{\mu}$ be the set of the distinct roots of (2.4), and let $\ell_{j}$ 's denote the multiplicity of $\alpha_{j}$. Then under the condition (ii) of Theorem 2.1, the Borel sum $u^{d}(t, x)$ for $(t, x) \in S(d, \beta, \rho) \times B_{r}$ with $\beta>\pi(q-p) / p$ and some $r>0$ is given by the following integral:

$$
\begin{aligned}
u^{d}(t, x)= & \left(\frac{t}{p}\right)^{p \nu-1} \sum_{j=1}^{\mu} \sum_{k=1}^{\ell_{j}} c_{j k} p^{1-k}\left(t \partial_{t}\right)^{k-1} \\
& \times \int_{0}^{\infty\left(\left(p d+\arg \alpha_{j}\right) / q\right)} \Phi(x, \zeta) \mathcal{K}_{\alpha_{j}}(t, \zeta) d \zeta,
\end{aligned}
$$

where

$$
\Phi(x, \zeta)=\sum_{m=0}^{q-1} \varphi\left(x+\zeta \omega_{q}^{m}\right), \quad \omega_{q}=e^{2 \pi i / q},
$$

and the functions $\mathcal{K}_{\alpha_{j}}(t, \zeta)(j=1,2, \ldots, \mu)$ are given by

$$
\mathcal{K}_{\alpha_{j}}(t, \zeta)=\frac{C_{p, q}}{\zeta} G_{p, q}^{q, 0}\left(\begin{array}{c|c}
Z_{\alpha_{j}} & \begin{array}{c}
\nu+\overline{\boldsymbol{p}} / p \\
\boldsymbol{q} / q
\end{array}
\end{array}\right),
$$

with

$$
Z_{\alpha_{j}}=\frac{p^{p}}{q^{q}} \frac{1}{\alpha_{j}} \frac{\zeta^{q}}{t^{p}}, \quad C_{p, q}=\frac{\boldsymbol{\Gamma}(\boldsymbol{p} / p)}{\boldsymbol{\Gamma}(\boldsymbol{q} / q)} .
$$

The $G$-function in the expression (2.27) is defined by the following integral along a path of integration $I=\{\tau \in \mathbb{C} ; \operatorname{Re} \tau=\kappa>-1 / q\}$

$$
G_{p, q}^{q, 0}\left(\begin{array}{c|c}
Z_{\alpha_{j}} & \begin{array}{c}
\nu+\overline{\boldsymbol{p}} / p \\
\boldsymbol{q} / q
\end{array}
\end{array}\right)=\frac{1}{2 \pi i} \int_{I} \frac{\boldsymbol{\Gamma}(\boldsymbol{q} / q+\tau)}{\boldsymbol{\Gamma}(\nu+\overline{\boldsymbol{p}} / p+\tau)} Z_{\alpha_{j}}^{-\tau} d \tau
$$




$$
Z_{\alpha_{j}}\left(=\frac{p^{p}}{q^{q}} \frac{1}{\alpha_{j}} \frac{\zeta^{q}}{t^{p}}\right) \in S(0,(q-p) \pi, \infty) .
$$

By calculating the residues of the integrand on the left side $(\operatorname{Re} \tau<\kappa)$ of the path $I$, we obtain the following proposition.

Proposition 2.4 (A relationship between $G$-function and ${ }_{p} F_{q-1}$ ).

$$
G_{p, q}^{q, 0}\left(\begin{array}{c|c}
Z_{\alpha_{j}} & \begin{array}{c}
\nu+\overline{\boldsymbol{p}} / p \\
\boldsymbol{q} / q
\end{array}
\end{array}\right)=F_{\alpha_{j}}(t, \zeta)-P_{\alpha_{j}}(t, \zeta),
$$

where

$$
\begin{aligned}
F_{\alpha_{j}}(t, \zeta)=\sum_{\ell=1}^{q-1} & C_{p, q, \nu}(\ell) Z_{\alpha_{j}}^{\ell / q} \\
& \quad \times{ }_{p} F_{q-1}\left(\begin{array}{c}
1+\ell / q-\nu-\overline{\boldsymbol{p}} / p \\
1+\ell / q-\widehat{\boldsymbol{q}}_{\ell} / q
\end{array} ;(-1)^{p-q} Z_{\alpha_{j}}\right),
\end{aligned}
$$

$$
\begin{aligned}
P_{\alpha_{j}}(t, \zeta) & =C_{p, q, \nu} \sum_{m=1}^{\nu-1} \frac{(1-\nu-\overline{\boldsymbol{p}} / p)_{m}}{\left(1-\widehat{\boldsymbol{q}_{q}} / q\right)_{m} \Gamma(m)}\left((-1)^{p-q} Z_{\alpha_{j}}\right)^{m}, \text { (Polynomial) } \\
C_{p, q, \nu}(\ell) & =\frac{\boldsymbol{\Gamma}\left(\widehat{\boldsymbol{q}_{\ell}} / q-\ell / q\right)}{\boldsymbol{\Gamma}(\nu+\overline{\boldsymbol{p}} / p-\ell / q)}, \quad C_{p, q, \nu}=\frac{\boldsymbol{\Gamma}\left(\widehat{\boldsymbol{q}_{q}} / q\right)}{\boldsymbol{\Gamma}(\nu+\overline{\boldsymbol{p}} / p)}
\end{aligned}
$$

Proposition 2.4 gives the following formula for the kernel functions $\mathcal{K}_{\alpha_{j}}(t, \zeta)$

$$
\mathcal{K}_{\alpha_{j}}(t, \zeta)=\frac{C_{p, q}}{\zeta}\left(F_{\alpha_{j}}(t, \zeta)-P_{\alpha_{j}}(t, \zeta)\right)
$$

In particular, in the case where the arguments of $\alpha_{j}$ are all the same, the polynomial parts $P_{\alpha_{j}}$ disappear from the functions $\mathcal{K}_{\alpha_{j}}$ in (2.34) and we have

Corollary 2.5. If $\arg \alpha_{j}=\theta$ for all $j$, then the Borel sum $u^{d}(t, x)$ is given by

$$
\begin{aligned}
u^{d}(t, x)= & \left(\frac{t}{p}\right)^{p \nu-1} \sum_{j=1}^{\mu} \sum_{k=1}^{\ell_{j}} c_{j k} p^{1-k}\left(t \partial_{t}\right)^{k-1} \\
& \times \int_{0}^{\infty((p d+\theta) / q)} \Phi(x, \zeta) \times \frac{C_{p, q}}{\zeta} F_{\alpha_{j}}(t, \zeta) d \zeta .
\end{aligned}
$$


We next consider the case where the characteristic equation (2.4) has only one root, that is, $\alpha_{j}=\alpha$ for all $j$. In this case the Cauchy problem (2.3) has a formal solution of the form (2.6)-(2.7) with

$$
A(n)=\alpha^{n} \sum_{j=1}^{\nu} c_{j} n^{j-1}=\frac{(n+1)(n+2) \cdots(n+\nu-1)}{(\nu-1) !} \alpha^{n}=\frac{(\nu)_{n}}{n !} \alpha^{n} .
$$

Then the Borel sum $u^{d}(t, x)$ is given by the following theorem.

Theorem 2.6. $\quad$ Suppose that the equation (2.4) has only one root $\alpha$ with $\nu$-multiplicity. Then under the condition (ii) of Theorem 2.1, the Borel sum $u^{d}(t, x)$ is given by

$$
u^{d}(t, x)=\left(\frac{t}{p}\right)^{p \nu-1} \int_{0}^{\infty((p d+\arg \alpha) / q)} \Phi(x, \zeta) K_{\alpha}(t, \zeta) d \zeta,
$$

where $\Phi(x, \zeta)$ is given by $(2.26)$ and the function $K_{\alpha}(t, \zeta)$ is given by

$$
\begin{aligned}
& K_{\alpha}(t, \zeta) \\
= & \frac{D_{p, q, \nu}}{\zeta} G_{p-1, q-1}^{q-1,0}\left(Z_{\alpha} \mid \begin{array}{c}
\nu+\widehat{\boldsymbol{p}_{p}} / p \\
\widehat{\boldsymbol{q}_{q}} / q
\end{array}\right) \\
= & \frac{D_{p, q, \nu}}{\zeta} \sum_{\ell=1}^{q-1} D_{p, q, \nu}(\ell) Z_{\alpha}^{\ell / q} \\
& \quad{ }_{p-1} F_{q-2}\left(\begin{array}{c}
1+\ell / q-\nu-\widehat{\boldsymbol{p}_{p}} / p \\
1+\ell / q-\widehat{\left(\widehat{\boldsymbol{q}_{q}}\right)} / q
\end{array} ;(-1)^{p-q} Z_{\alpha}\right),
\end{aligned}
$$

with $Z_{\alpha}=\left(p^{p} / q^{q}\right)(1 / \alpha)\left(\zeta^{q} / t^{p}\right)$ and

$$
D_{p, q, \nu}=\frac{\boldsymbol{\Gamma}(\boldsymbol{p} / p)}{\Gamma(\nu) \boldsymbol{\Gamma}(\boldsymbol{q} / q)}, \quad D_{p, q, \nu}(\ell)=\frac{\boldsymbol{\Gamma}\left(\widehat{\left(\widehat{\boldsymbol{q}_{q}}\right)} / q-\ell / q\right)}{\boldsymbol{\Gamma}\left(\nu+\widehat{\boldsymbol{p}_{p}} / p-\ell / q\right)} .
$$

If $p=1$ or $q=2$, then $\widehat{\boldsymbol{p}_{p}}$ or $\widehat{\left(\widehat{\boldsymbol{q}_{q}}\right)_{\ell}}$ are empty, respectively.

In the paper [Ich] we discussed the following Cauchy problem, corresponding to the case $\nu=1$ (therefore $\mu=1$ ) in our present setting.

$$
\left\{\begin{array}{l}
\partial_{t}^{p} u(t, x)=\alpha \partial_{x}^{q} u(t, x) \\
u(0, x)=\varphi(x), \quad \partial_{t}^{k} u(0, x)=0(k=0,1, \ldots, p-1) .
\end{array}\right.
$$


In [Ich] it is verified that under the condition (ii) of Theorem 2.1 the Borel sum $u^{d}(t, x)$ has the following representation:

$$
u^{d}(t, x)=\int_{0}^{\infty((p d+\arg \alpha) / q)} \Phi(x, \zeta) k_{\alpha}(t, \zeta) d \zeta,
$$

where the kernel function $k_{\alpha}(t, \zeta)$ is given by

$$
\begin{aligned}
k_{\alpha}(t, \zeta) & =\frac{C_{p, q}}{\zeta} \sum_{\ell=1}^{q-1} \widetilde{C}_{p, q}(\ell) Z_{\alpha}^{\ell / q}{ }_{p} F_{q-1}\left(\begin{array}{c}
1+\ell / q-\boldsymbol{p} / p \\
1+\ell / q-\widehat{\boldsymbol{q}}_{\ell} / q
\end{array} ;(-1)^{p-q} Z_{\alpha}\right) \\
& =\frac{C_{p, q}}{\zeta} G_{p, q}^{q, 0}\left(Z_{\alpha} \begin{array}{c}
\boldsymbol{p} / p \\
\boldsymbol{q} / q
\end{array}\right),
\end{aligned}
$$

with the same constant $C_{p, q}$ as in (2.28) and

$$
\widetilde{C}_{p, q}(\ell)=\frac{\boldsymbol{\Gamma}\left(\widehat{\boldsymbol{q}}_{\ell} / q-\ell / q\right)}{\boldsymbol{\Gamma}(\boldsymbol{p} / p-\ell / q)}
$$

Comparing this result with Theorem 2.6 (for $\nu=1$ ), we find that there is the following relation between the kernel function $(t / p)^{p \nu-1} K_{\alpha}(t, \zeta)$ in Theorem 2.6 and $k_{\alpha}(t, \zeta)$ in $(2.42)$.

$$
\partial_{t}^{p-1}\left[\left.\left(\frac{t}{p}\right)^{p \nu-1} K_{\alpha}(t, \zeta)\right|_{\nu=1}\right]=k_{\alpha}(t, \zeta),
$$

that is,

$$
\left.\left(\frac{t}{p}\right)^{p \nu-1} K_{\alpha}(t, \zeta)\right|_{\nu=1}=\left(D_{t}^{-1}\right)^{p-1} k_{\alpha}(t, \zeta) .
$$

We can generalize this relation for general $\nu$ as follows.

Corollary 2.7. Let $\mathcal{K}_{\alpha}(t, \zeta)$ and $k_{\alpha}(t, \zeta)$ be the functions given in (2.27) and (2.42), respectively. For any fixed $\zeta$ with $\zeta \neq 0$, we have

$$
\left(\frac{t}{p}\right)^{p \nu-1} \mathcal{K}_{\alpha}(t, \zeta)=\left(D_{t}^{-1}\right)^{p \nu-1} k_{\alpha}(t, \zeta),
$$

where $D_{t}^{-1}$ denotes the integration from 0 to $t$. 


\section{§2.12. Example}

In order to illustrate our results, let us examine the following Cauchy problem.

$$
\left\{\begin{array}{l}
\prod_{j=1}^{2}\left(\partial_{t}-\alpha_{j} \partial_{x}^{2}\right) u(t, x)=0, \\
u(0, x)=0, \quad \partial_{t} u(0, x)=\varphi(x)
\end{array}\right.
$$

where $\alpha_{j} \in \mathbb{C} \backslash\{0\}(j=1,2)$ and we assume that $\varphi(x)$ satisfies the conditions for the Borel summability in $d$ direction.

When $\arg \alpha_{1} \neq \arg \alpha_{2}$, the Borel sum $u^{d}(t, x)$ is given by

$$
\begin{aligned}
& u^{d}(t, x)=\sum_{j=1}^{2} c_{j} \int_{0}^{\infty\left(\left(d+\arg \alpha_{j}\right) / 2\right)} \Phi(x, \zeta) \\
& \times\left\{\sqrt{\frac{t}{\pi \alpha_{j}}}{ }_{1} F_{1}\left(\begin{array}{c}
-1 / 2 \\
1 / 2
\end{array} ;-\frac{\zeta^{2}}{4 \alpha_{j} t}\right)-\frac{\zeta}{2 \alpha_{j}}\right\} d \zeta \\
& =\sum_{j=1}^{2} c_{j} \int_{0}^{\infty\left(\left(d+\arg \alpha_{j}\right) / 2\right)} \Phi(x, \zeta) \\
& \times\left\{\int_{0}^{t} \frac{1}{\sqrt{4 \alpha_{j} \pi \tau}} \exp \left(-\frac{\zeta^{2}}{4 \alpha_{j} \tau}\right) d \tau\right\} d \zeta \\
& =\sum_{j=1}^{2} c_{j} \int_{e^{i \psi_{j}} \mathbb{R}} \varphi(x+\zeta) \\
& \times\left\{\int_{0}^{t} \frac{1}{\sqrt{4 \alpha_{j} \pi \tau}} \exp \left(-\frac{\zeta^{2}}{4 \alpha_{j} \tau}\right) d \tau\right\} d \zeta,
\end{aligned}
$$

where $e^{i \psi_{j}} \mathbb{R}=\left\{r e^{i \psi_{j}} ;-\infty<r<\infty, \psi_{j}=\left(d+\arg \alpha_{j}\right) / 2\right\}$ and

$$
c_{1}=\frac{\alpha_{1}}{\alpha_{1}-\alpha_{2}}, \quad c_{2}=\frac{\alpha_{2}}{\alpha_{2}-\alpha_{1}} .
$$

Here the first equality is obtained by the formulas (2.25) and (2.30), the second equality is obtained by the formula (2.45) and the last equality is obtained by representing the second one with integrations along a line.

When $\arg \alpha_{1}=\arg \alpha_{2}=\theta \quad\left(\alpha_{1} \neq \alpha_{2}\right)$, we have

$$
u^{d}(t, x)=\int_{e^{i \psi \mathbb{R}}} \varphi(x+\zeta)\left\{\sum_{j=1}^{2} c_{j} \sqrt{\frac{t}{\pi \alpha_{j}}}{ }_{1} F_{1}\left(\begin{array}{c}
-1 / 2 \\
1 / 2
\end{array} ;-\frac{\zeta^{2}}{4 \alpha_{j} t}\right)\right\} d \zeta
$$


where $\psi=(d+\theta) / 2$. This expression is obtained from Corollary 2.5, or by noting that $c_{1} / \alpha_{1}+c_{2} / \alpha_{2}=0$ in the first equality of (2.47).

When $\alpha_{1}=\alpha_{2}=\alpha$, we have

$$
u^{d}(t, x)=\int_{e^{i \psi \mathbb{R}}} \varphi(x+\zeta) \sqrt{\frac{t}{4 \alpha \pi}} \exp \left(-\frac{\zeta^{2}}{4 \alpha t}\right) d \zeta,
$$

where $\psi=(d+\arg \alpha) / 2$. This expression is derived from (2.48) by taking a limit $\alpha_{2} \rightarrow \alpha_{1}(=\alpha)$.

\section{§3. $\quad$ Proof of Theorems 2.1 and 2.3}

It is sufficient to prove the equivalence between (i) and (ii). For this purpose, we use the following important lemma for the Borel summability (cf. [Bal 1], [LMS], [Miy 3]).

Lemma 3.1. Let $k>0, d \in \mathbb{R}$ and $\hat{u}(t, x) \in \mathcal{O}[[t]]_{1 / k}$. Then the following three statements are equivalent:

(i) $\hat{u}(t, x)$ is $k$-summable in d direction.

(ii) Let $v_{1}(s, x)$ be the formal $k$-Borel transform of $\hat{u}(t, x)$

$$
v_{1}(s, x)=\left(\hat{\mathcal{B}}_{k} \hat{u}\right)(s, x):=\sum_{n=0}^{\infty} u_{n}(x) \frac{s^{n}}{\Gamma(1+n / k)},
$$

which is holomorphic in a neighbourhood of the origin. Then $v_{1}(s, x)$ can be continued analytically in a sector $S(d, \varepsilon, \infty)$ in s-plane for some positive constant $\varepsilon$ and satisfies a growth condition of exponential order at most $k$ there, that is, there exist some positive constants $C$ and $\gamma$ such that

$$
\max _{|x| \leq r}\left|v_{1}(s, x)\right| \leq C \exp \left\{\gamma|s|^{k}\right\}, \quad s \in S(d, \varepsilon, \infty) .
$$

(iii) Let $j \geq 2$ and $k_{1}>0, \ldots, k_{j}>0$ satisfy $1 / k=1 / k_{1}+\cdots+1 / k_{j}$. Let $v_{2}(s, x)$ be the following iterated formal Borel transform of $\hat{u}(t, x)$

$$
v_{2}(s, x)=\left(\hat{\mathcal{B}}_{k_{j}} \circ \cdots \circ \hat{\mathcal{B}}_{k_{1}} \hat{u}\right)(s, x) .
$$

Then $v_{2}(s, x)$ has the same properties as $v_{1}(s, x)$ above. 
Under these conditions, the Borel sum $u^{d}(t, x)$ is given by the following $k$ Laplace integral, where the integration is done along the half line of argument $d$.

$$
u^{d}(t, x)=\left(\mathcal{L}_{k} v_{1}\right)(t, x):=\frac{1}{t^{k}} \int_{0}^{\infty(d)} \exp \left[-\left(\frac{s}{t}\right)^{k}\right] v_{1}(s, x) d\left(s^{k}\right),
$$

where $(t, x) \in S(d, \beta, \rho) \times B_{r}$ with $\beta<\pi / k$ and $r>0$. The Borel sum $u^{d}(t, x)$ is also obtained as the following iterated Laplace integral

$$
u^{d}(t, x)=\left(\mathcal{L}_{k_{1}} \circ \cdots \circ \mathcal{L}_{k_{j}} v_{2}\right)(t, x) .
$$

Proof of Theorem 2.1. Suppose that the Cauchy data $\varphi(x)$ satisfies the condition (ii) of Theorem 2.1. Let $v(s, x)$ be the $(q-p)$ times iterated formal $p$-Borel transform of $\hat{u}(t, x)$ which is given by $(2.6)$

$$
\begin{aligned}
v(s, x) & =\left(\hat{\mathcal{B}}_{p}^{q-p} \hat{u}\right)(s, x) \\
& =\sum_{n \geq 0} \frac{u_{p n+p \nu-1}(x)}{(p n+p \nu-1) !} \frac{s^{p n+p \nu-1}}{\Gamma(1+(p n+p \nu-1) / p)^{q-p}} .
\end{aligned}
$$

By using (2.7) and (2.10), we have

$$
\begin{aligned}
v(s, x) & =s^{p \nu-1} \sum_{n \geq 0} \frac{A(n) \varphi^{(q n)}(x)}{(p n+p \nu-1) !} \frac{s^{p n}}{\Gamma(1+(p n+p \nu-1) / p)^{q-p}} \\
& =s^{p \nu-1} \sum_{j=1}^{\mu} \sum_{k=1}^{\ell_{j}} c_{j k} \sum_{n \geq 0} \frac{\varphi^{(q n)}(x) n^{k-1} \alpha_{j}^{n} s^{p n}}{(p n+p \nu-1) ! \Gamma(1+(p n+p \nu-1) / p)^{q-p}}
\end{aligned}
$$

From the Cauchy integral formula for some sufficiently small $|s|$ and $|x|$, we have

$$
\begin{aligned}
v(s, x)= & \frac{s^{p \nu-1}}{2 \pi i} \sum_{j=1}^{\mu} \sum_{k=1}^{\ell_{j}} c_{j k} \oint_{|\zeta|=r} \frac{\varphi(x+\zeta)}{\zeta} \\
& \times \sum_{n \geq 0} \frac{n^{k-1}(q n) ! \alpha_{j}^{n}}{(p n+p \nu-1) ! \Gamma(1+(p n+p \nu-1) / p)^{q-p}} \frac{s^{p n}}{\zeta^{q n}} d \zeta \\
= & \frac{s^{p \nu-1}}{2 \pi i} \sum_{j=1}^{\mu} \sum_{k=1}^{\ell_{j}} c_{j k} \oint_{|\zeta|=r} \frac{\varphi(x+\zeta)}{\zeta} h_{j k}(s, \zeta) d \zeta,
\end{aligned}
$$


where

$$
h_{j k}(s, \zeta)=\sum_{n \geq 0} \frac{n^{k-1} \cdot(q n) !}{(p n+p \nu-1) ! \Gamma(1+(p n+p \nu-1) / p)^{q-p}}\left(\frac{\alpha_{j} s^{p}}{\zeta^{q}}\right)^{n}
$$

and $\max \left\{\left|q^{q} \alpha_{j} s^{p} / p^{p}\right|^{1 / q}\right\}<r$ (cf. (3.11) below).

We recall the notation $(c)_{n}=\Gamma(c+n) / \Gamma(c)$ and the multiplication formula of the Gamma function for $m=2,3, \ldots$

$$
\Gamma(m z)=(2 \pi)^{-(m-1) / 2} m^{m z-1 / 2} \boldsymbol{\Gamma}\left(z+\frac{\overline{\boldsymbol{m}}}{m}\right),
$$

where $z+k / m \notin \mathbb{Z}_{\leq 0}(k=0,1, \ldots, m-1)$ (cf. [Erd, p.4]). Then we have $(q n) !=q^{q n}(\boldsymbol{q} / q)_{n}$ and

$$
\begin{aligned}
(p n+p \nu-1) ! & =\Gamma(p n+p \nu)=\Gamma(p(n+\nu)) \\
& =(2 \pi)^{-(p-1) / 2} p^{p(n+\nu)-1 / 2} \boldsymbol{\Gamma}(n+\nu+\overline{\boldsymbol{p}} / p) \\
& =p^{p n}(2 \pi)^{-(p-1) / 2} p^{p \nu-1 / 2} \boldsymbol{\Gamma}(\nu+\overline{\boldsymbol{p}} / p)(\nu+\overline{\boldsymbol{p}} / p)_{n} \\
& =p^{p n} \Gamma(p \nu)(\nu+\overline{\boldsymbol{p}} / p)_{n} .
\end{aligned}
$$

We denote $h_{j 1}(s, \zeta)$ by $h_{\alpha_{j}}(s, \zeta)$. Then we have

$$
\begin{aligned}
h_{\alpha_{j}}(s, \zeta) & =\sum_{n \geq 0} \frac{(q n) !}{(p n+p \nu-1) ! \Gamma(1+(p n+p \nu-1) / p)^{q-p}}\left(\frac{\alpha_{j} s^{p}}{\zeta^{q}}\right)^{n} \\
& =\frac{1}{C_{0}} q+1 F_{q}\left(\begin{array}{c}
\boldsymbol{q} / q, 1 \\
\left.\nu+\overline{\boldsymbol{p}} / p, \nu+\frac{p-1}{p}, \ldots, \nu+\frac{p-1}{p} ; \frac{q^{q}}{p^{p}} \cdot \frac{\alpha_{j} s^{p}}{\zeta^{q}}\right)
\end{array}\right.
\end{aligned}
$$

where $C_{0}=\Gamma(p \nu) \Gamma(\nu+(p-1) / p)^{q-p}$, and $h_{j k}(s, \zeta)=p^{1-k}\left(s \partial_{s}\right)^{k-1} h_{\alpha_{j}}(s, \zeta)$ for $k=1,2, \ldots, \ell_{j}$. Therefore we obtain

$$
\begin{aligned}
v(s, x) & =\frac{s^{p \nu-1}}{2 \pi i} \sum_{j=1}^{\mu} \sum_{k=1}^{\ell_{j}} c_{j k} p^{1-k}\left(s \partial_{s}\right)^{k-1} \oint_{|\zeta|=r} \frac{\varphi(x+\zeta)}{\zeta} h_{\alpha_{j}}(s, \zeta) d \zeta . \\
& =\frac{s^{p \nu-1}}{2 \pi i} \sum_{j=1}^{\mu} \sum_{k=1}^{\ell_{j}} c_{j k} p^{1-k}\left(s \partial_{s}\right)^{k-1} I_{\alpha_{j}}(s, x)
\end{aligned}
$$

where

$$
I_{\alpha_{j}}(s, x)=\oint_{|\zeta|=r} \frac{\varphi(x+\zeta)}{\zeta} h_{\alpha_{j}}(s, \zeta) d \zeta .
$$

From this expression, we can prove that under the conditions for the Cauchy data $\varphi(x)$, each $I_{\alpha_{j}}(s, x)$ can be continued analytically in a sector 
$S(d, \varepsilon, \infty)$ on $s$-space for some positive constant $\varepsilon$ and has the growth condition of exponential order at most $q /(q-p)$ there. In fact, these follow from the fact that ${ }_{q+1} F_{q}(\boldsymbol{\beta} ; \boldsymbol{\gamma} ; z)$ is a holomorphic solution at $z=0$ of a Fuchsian ordinary differential equation

$$
E_{q+1, q}[\boldsymbol{\beta} ; \boldsymbol{\gamma}](f):=\left[\prod_{j=1}^{q} \delta_{z}\left(\delta_{z}+\gamma_{j}-1\right)-z \prod_{\ell=1}^{q+1}\left(\delta_{z}+\beta_{\ell}\right)\right] f(z)=0
$$

which has three singular points at $z=0,1, \infty$. This observation proves that (ii) implies (i) (cf. [LMS], [Miy 3]).

Conversely, suppose the condition (i) of Theorem 2.1, that is, we suppose that $\hat{u}(t, x)$ is $p /(q-p)$-summable in $d$ direction.

For any fixed $j(j=1,2, \ldots, \mu)$, let

$$
Q_{j}=\prod_{k=1, k \neq j}^{\mu} P_{k}^{\ell_{k}} \cdot P_{j}^{\ell_{j}-1},
$$

and put $\hat{w}_{j}(t, x):=Q_{j} \hat{u}(t, x)$. Then the assumption that $\hat{u}(t, x)$ is $p /(q-p)$ summable in $d$ direction implies that $\hat{w}_{j}(t, x)$ is also $p /(q-p)$-summable in the same direction. In fact, $Q_{j} u^{d}(t, x)$ gives the Borel sum of $\hat{w}_{j}(t, x)$ in $d$ direction.

On the other hand, $\hat{f}_{j}(t, x):=\partial_{t}^{p-1} \hat{w}_{j}(t, x)$, which is also $p /(q-p)$ summable in $d$ direction, is a formal solution of the following Cauchy problem

$$
\left(E_{j}\right) \quad\left\{\begin{array}{l}
\left(\partial_{t}^{p}-\alpha_{j} \partial_{x}^{q}\right) \hat{f}_{j}(t, x)=0 \\
\hat{f}_{j}(0, x)=\varphi(x) \\
\partial_{t}^{k} \hat{f}_{j}(0, x)=0(1 \leq k \leq p-1) .
\end{array}\right.
$$

By the result in [Miy 3], we already know that $\hat{f}_{j}(t, x)$ is $p /(q-p)$-summable in $d$ direction if and only if the Cauchy data $\varphi(x)$ can be continued analytically in $\Omega_{\alpha_{j}}$ and satisfies the growth condition of exponential order at most $q /(q-p)$ there. This implies the statement (ii) for the Cauchy data $\varphi(x)$.

Proof of Theorem 2.3. In the expression (3.12), we notice that for each $j(j=1,2, \ldots, \mu) h_{\alpha_{j}}(s, \zeta)$ has $q$ singular points in $\zeta$-plane at $q$ roots of $\zeta^{q}=\left(q^{q} / p^{p}\right) \alpha_{j} s^{p}$. We put $\zeta_{j}(s)=\left(q^{q} / p^{p}\right)^{1 / q}\left(\alpha_{j} s^{p}\right)^{1 / q}$ with $\arg \zeta_{j}(s)=(d p+$ $\left.\arg \alpha_{j}\right) / q$ for a fixed $s \neq 0$ and $\arg s=d$, and we denote by $\left[0, \zeta_{j}(s)\right]$ the segment joining the origin and $\zeta_{j}(s)$. Then $h_{\alpha_{j}}(s, \zeta)$ is univalent in $\mathbb{C}_{\zeta} \backslash \cup_{m=0}^{q-1}\left[0, \zeta_{j}(s) \omega_{q}^{m}\right]$ and we note that the integrand is integrable at $\zeta=0$ and $\zeta=\zeta_{j}(s)$. In fact, we notice that $w(z)=h_{\alpha}(s, \zeta)$ with $z=\left(q^{q} \alpha s^{p}\right) /\left(p^{p} \zeta^{q}\right)$ satisfies

$$
E_{q+1, q}[\boldsymbol{q} / q, 1 ; \nu+\overline{\boldsymbol{p}} / p, \nu+(p-1) / p, \ldots, \nu+(p-1) / p](w)=0 .
$$


The characteristic exponents at $z=1$ are $\{0,1,2, \ldots, q-1, \lambda\}$ where

$$
\begin{aligned}
\lambda & =\sum_{\ell=0}^{p-1}\left(\nu+\frac{\ell}{p}\right)+(q-p)\left(\nu+\frac{p-1}{p}\right)-\sum_{m=1}^{q} \frac{m}{q}-1 \\
& =(q-p)\left(\frac{p-1}{p}-\frac{1}{2}\right)+\nu q-2(>-1),
\end{aligned}
$$

which implies the integrability at $\zeta=\zeta_{j}(s)$, and the characteristic exponents at $z=\infty$ are $\{1 / q, 2 / q, \ldots, 1,1\}$, which implies the integrability at $\zeta=0$. Therefore by the assumption that $\varphi(x)$ is analytic in $\Omega_{x}$, we can deform the path of integration of $I_{\alpha_{j}}(s, x)$ as follows (cf. [Ich]).

$$
I_{\alpha_{j}}(s, x)=\int_{0}^{\zeta_{j}(s)} \frac{\Phi(x, \zeta)}{\zeta} H_{\alpha_{j}}(s, \zeta) d \zeta
$$

where $\Phi(x, \zeta)$ is given by $(2.26)$ and

$$
H_{\alpha_{j}}(s, \zeta)=h_{\alpha_{j}}(s, \zeta)-h_{\alpha_{j}}\left(s, \omega_{q}^{-1} \zeta\right) .
$$

Moreover, since each $h_{\alpha_{j}}(s, \zeta)$ is univalent outside of the $q$ segments $\left[0, \zeta_{j}(s) \omega_{q}^{m}\right]$, we have

$$
I_{\alpha_{j}}(s, x)=\int_{0}^{\infty\left(\left(p d+\arg \alpha_{j}\right) / q\right)} \frac{\Phi(x, \zeta)}{\zeta} H_{\alpha_{j}}(s, \zeta) d \zeta .
$$

Therefore the Borel sum $u^{d}(t, x)$ is given by the following $(q-p)$ times iterated $p$-Laplace integral

$$
\begin{aligned}
u^{d}(t, x)= & \left(\mathcal{L}_{p}^{q-p} v\right)(t, x) \\
= & {\left[\mathcal { L } _ { p } ^ { q - p } \left(\frac{s^{p \nu-1}}{2 \pi i} \sum_{j=1}^{\mu} \sum_{k=1}^{\ell_{j}} c_{j k} p^{1-k}\left(s \partial_{s}\right)^{k-1}\right.\right.} \\
& \left.\left.\times \int_{0}^{\infty\left(\left(p d+\arg \alpha_{j}\right) / q\right)} \frac{\Phi(x, \zeta)}{\zeta} H_{\alpha_{j}}(s, \zeta) d \zeta\right)\right](t, x) .
\end{aligned}
$$

By exchanging the order of integrations, we have

$$
\begin{aligned}
u^{d}(t, x)= & \sum_{j=1}^{\mu} \sum_{k=1}^{\ell_{j}} c_{j k} \int_{0}^{\infty\left(\left(p d+\arg \alpha_{j}\right) / q\right)} \Phi(x, \zeta) \\
& \times \frac{1}{2 \pi i \zeta}\left(\mathcal{L}_{p}^{q-p}\left(s^{p \nu-1} p^{1-k}\left(s \partial_{s}\right)^{k-1} H_{\alpha_{j}}(s, \zeta)\right)\right)(t, \zeta) d \zeta .
\end{aligned}
$$


We recall the following Barnes type integral representation of $h_{\alpha_{j}}(s, \zeta)$ (cf. [Luk], [IKSY])

$$
\begin{aligned}
& h_{\alpha_{j}}(s, \zeta)=\frac{1}{C_{0}} \\
& \times_{q+1} F_{q}\left(\begin{array}{c}
\boldsymbol{q} / q, 1 \\
\left.\nu+\overline{\boldsymbol{p}} / p, \nu+\frac{p-1}{p}, \ldots, \nu+\frac{p-1}{p} ; \frac{q^{q}}{p^{p}} \cdot \frac{\alpha_{j} s^{p}}{\zeta^{q}}\right)
\end{array}\right. \\
& =\frac{C_{1}}{2 \pi i C_{0}} \int_{I} \frac{\boldsymbol{\Gamma}(\boldsymbol{q} / q+\tau) \Gamma(1+\tau) \Gamma(-\tau)}{\boldsymbol{\Gamma}(\nu+\overline{\boldsymbol{p}} / p+\tau) \Gamma(\nu+(p-1) / p+\tau)^{q-p}} \\
& \times\left(-\alpha_{j} \frac{q^{q} s^{p}}{p^{p} \zeta^{q}}\right)^{\tau} d \tau,
\end{aligned}
$$

where $\left|\arg \left(-z_{j}\right)\right|<\pi$ with $z_{j}=\left(q^{q} \alpha_{j} s^{p}\right) /\left(p^{p} \zeta^{q}\right)$ and $\left(-z_{j}\right)^{\tau}=\exp \left(\tau \log \left(-z_{j}\right)\right)$ $=\exp \left\{\tau\left(\log \left|z_{j}\right|+i\left(\arg z_{j}-\pi\right)\right)\right\}$, the path of integration $I$ runs from $\kappa-i \infty$ to $\kappa+i \infty$ with $-1 / q<\kappa<0$ and

$$
C_{1}=\frac{\Gamma(\nu+(p-1) / p)^{q-p} \boldsymbol{\Gamma}(\nu+\overline{\boldsymbol{p}} / p)}{\boldsymbol{\Gamma}(\boldsymbol{q} / q)} .
$$

Here we notice that by consulting (2.22) and (2.23) we have that if $\left|\arg \left(-z_{j}\right)\right| \leq$ $\pi$, the above integral converges absolutely when

$$
(q-p)\left(\frac{p-1}{q}-\frac{1}{2}\right)+q \nu-2>0 .
$$

Therefore the above integral (3.23) converges absolutely in $\left|\arg \left(-z_{j}\right)\right| \leq \pi$ except the cases $(p, q, \nu)=(1,2,1)$ and $(1,3,1)$.

In the below, we prove the theorem in the cases when $(p, q, \nu) \neq(1,2,1)$, $(1,3,1)$ and the case $(p, q, \nu)=(1,3,1)$ will be proved in Appendix since we need a different proof, which will be done by employing the Euler type integral representation of $h_{\alpha_{j}}(s, \zeta)$. (The case $(p, q, \nu)=(1,2,1)$ was studied by [LMS].)

Now, since the function $H_{\alpha_{j}}(s, \zeta)$ is well-defined on the line of $\arg \zeta=$ $\left(p d+\arg \alpha_{j}\right) / q$ for a fixed $s \neq 0$ with $\arg s=d$, we have

$$
\begin{aligned}
H_{\alpha_{j}}(s, \zeta)= & h_{\alpha_{j}}(s, \zeta)-h_{\alpha_{j}}\left(s, \omega_{q}^{-1} \zeta\right) \\
= & \frac{C_{1}}{2 \pi i C_{0}} \int_{I} \frac{\boldsymbol{\Gamma}(\boldsymbol{q} / q+\tau) \Gamma(1+\tau) \Gamma(-\tau)}{\boldsymbol{\Gamma}(\nu+\overline{\boldsymbol{p}} / p+\tau) \Gamma(\nu+(p-1) / p+\tau)^{q-p}} \\
& \times\left(-\alpha_{j} \frac{q^{q} s^{p}}{p^{p} \zeta^{q}}\right)^{\tau}\left(1-e^{2 \pi i \tau}\right) d \tau \\
= & \frac{C_{1}}{2 \pi i C_{0}} \int_{I} \frac{\boldsymbol{\Gamma}(\boldsymbol{q} / q+\tau) \Gamma(1+\tau) \Gamma(-\tau)}{\boldsymbol{\Gamma}(\nu+\overline{\boldsymbol{p}} / p+\tau) \Gamma(\nu+(p-1) / p+\tau)^{q-p}} \\
& \times\left(\alpha_{j} \frac{q^{q} s^{p}}{p^{p} \zeta^{q}}\right)^{\tau}(-2 i) \sin (\pi \tau) d \tau .
\end{aligned}
$$


By using the reflection formula of the Gamma function

$$
\sin \pi \tau=\frac{\pi}{\Gamma(\tau) \Gamma(1-\tau)},
$$

we have $\Gamma(1+\tau) \Gamma(-\tau) \sin (-\pi \tau)=\pi$, and hence

$$
\begin{aligned}
H_{\alpha_{j}}(s, \zeta)= & \frac{C_{1}}{C_{0}} \int_{I} \frac{\boldsymbol{\Gamma}(\boldsymbol{q} / q+\tau)}{\boldsymbol{\Gamma}(\nu+\overline{\boldsymbol{p}} / p+\tau) \Gamma(\nu+(p-1) / p+\tau)^{q-p}} \\
& \times\left(\alpha_{j} \frac{q^{q} s^{p}}{p^{p} \zeta^{q}}\right)^{\tau} d \tau .
\end{aligned}
$$

By substituting the integral representation (3.28) of $H_{\alpha_{j}}$, we calculate the iterated Laplace integral carefully as follows.

$$
\begin{aligned}
& \left(\mathcal{L}_{p}^{q-p}\left(s^{p \nu-1} p^{1-k}\left(s \partial_{s}\right)^{k-1} H_{\alpha_{j}}(s, \zeta)\right)\right)(t, \zeta) \\
= & \frac{C_{1}}{C_{0}}\left[\mathcal { L } _ { p } ^ { q - p } \left(\int_{I} \frac{\boldsymbol{\Gamma}(\boldsymbol{q} / q+\tau) \tau^{k-1} s^{p \nu+p \tau-1}}{\boldsymbol{\Gamma}(\nu+\overline{\boldsymbol{p}} / p+\tau) \Gamma(\nu+(p-1) / p+\tau)^{q-p}}\right.\right. \\
& \left.\left.\times\left(\alpha_{j} \frac{q^{q}}{p^{p} \zeta^{q}}\right)^{\tau} d \tau\right)\right](t, \zeta) \\
= & \frac{C_{1}}{C_{0}} \int_{I} \frac{\boldsymbol{\Gamma}(\boldsymbol{q} / q+\tau) \tau^{k-1}}{\boldsymbol{\Gamma}(\nu+\overline{\boldsymbol{p}} / p+\tau) \Gamma(\nu+(p-1) / p+\tau)^{q-p}}\left(\alpha_{j} \frac{q^{q}}{p^{p} \zeta^{q}}\right)^{\tau} d \tau \\
& \times\left[\mathcal{L}_{p}^{q-p} s^{p \nu+p \tau-1}\right](t) \\
= & \frac{C_{1}}{C_{0}} t^{p \nu-1} \int_{I} \frac{\boldsymbol{\Gamma}(\boldsymbol{q} / q+\tau) \tau^{k-1}}{\boldsymbol{\Gamma}(\nu+\overline{\boldsymbol{p}} / p+\tau)}\left(\alpha_{j} \frac{q^{q} t^{p}}{p^{p} \zeta^{q}}\right)^{\tau} d \tau \\
= & \frac{C_{1}}{C_{0}} t^{p \nu-1} p^{1-k}\left(t \partial_{t}\right)^{k-1} \int_{I} \frac{\boldsymbol{\Gamma}(\boldsymbol{q} / q+\tau)}{\boldsymbol{\Gamma}(\nu+\overline{\boldsymbol{p}} / p+\tau)}\left(\alpha_{j} \frac{q^{q} t^{p}}{p^{p} \zeta^{q}}\right)^{\tau} d \tau \\
= & 2 \pi i \frac{C_{1}}{C_{0}} t^{p \nu-1} p^{1-k}\left(t \partial_{t}\right)^{k-1} G_{p, q}^{q, 0}\left(Z_{\alpha_{j}} \mid \begin{array}{c}
\boldsymbol{q} / q \\
\overline{\boldsymbol{p}} / p
\end{array}\right) .
\end{aligned}
$$

Finally, let us calculate the constant

$$
\frac{C_{1}}{C_{0}}=\frac{\boldsymbol{\Gamma}(\nu+\overline{\boldsymbol{p}} / p)}{\Gamma(p \nu) \boldsymbol{\Gamma}(\boldsymbol{q} / q)} .
$$

Since $\boldsymbol{\Gamma}(\nu+\overline{\boldsymbol{p}} / p)=(2 \pi)^{(p-1) / 2} p^{1 / 2-p \nu} \Gamma(p \nu)$ and $\boldsymbol{\Gamma}(\boldsymbol{p} / p)=(2 \pi)^{(p-1) / 2} p^{-1 / 2}$ hold due to the multiplication formula of the Gamma function (3.10), we have

$$
\frac{C_{1}}{C_{0}}=\frac{\boldsymbol{\Gamma}(\boldsymbol{p} / p)}{\boldsymbol{\Gamma}(\boldsymbol{q} / q) p^{p \nu-1}} .
$$

Summing up the above considerations, we get the desired formula (2.25). 


\section{$\S 4$. Proof of Proposition 2.4}

The $G$-function in the expression (2.27) has the following integral representation by a path of integration $I=\{\tau \in \mathbb{C} \mid \operatorname{Re} \tau=\kappa>-1 / q\}$

$$
G_{p, q}^{q, 0}\left(\begin{array}{c|c}
Z_{\alpha_{j}} & \begin{array}{c}
\nu+\overline{\boldsymbol{p}} / p \\
\boldsymbol{q} / q
\end{array}
\end{array}\right)=\frac{1}{2 \pi i} \int_{I} \frac{\boldsymbol{\Gamma}(\boldsymbol{q} / q+\tau)}{\boldsymbol{\Gamma}(\nu+\overline{\boldsymbol{p}} / p+\tau)} Z_{\alpha_{j}}^{-\tau} d \tau, \quad Z_{\alpha_{j}}=\frac{p^{p}}{q^{q}} \frac{1}{\alpha_{j}} \frac{\zeta^{q}}{t^{p}}
$$

For the integrand, by using the formula $\Gamma(1+z)=z \Gamma(z)$, we have

$$
\frac{\boldsymbol{\Gamma}(\boldsymbol{q} / q+\tau) Z_{\alpha_{j}}^{-\tau}}{\boldsymbol{\Gamma}(\nu+\overline{\boldsymbol{p}} / p+\tau)}=\frac{\boldsymbol{\Gamma}\left(\widehat{\boldsymbol{q}_{q}} / q+\tau\right) Z_{\alpha_{j}}^{-\tau}}{\boldsymbol{\Gamma}\left(\nu+\widehat{\boldsymbol{p}_{p}} / p+\tau\right)} \times \frac{1}{(\nu-1+\tau)(\nu-2+\tau) \cdots(1+\tau)} .
$$

By calculating the residues of the integrand on the left side of the path $I$, we have

$$
\begin{aligned}
& G_{p, q}^{q, 0}\left(\begin{array}{c|c}
Z_{\alpha_{j}} & \begin{array}{c}
\nu+\overline{\boldsymbol{p}} / p \\
\boldsymbol{q} / q
\end{array}
\end{array}\right) \\
& =\sum_{\ell=1}^{q-1} \sum_{n \geq 0} \frac{(-1)^{n}}{n !} \frac{\boldsymbol{\Gamma}\left(\widehat{\boldsymbol{q}}_{\ell} / q-\ell / q-n\right)}{\boldsymbol{\Gamma}(\nu+\overline{\boldsymbol{p}} / p-\ell / q-n)} Z_{\alpha_{j}}^{\ell / q+n} \\
& +\sum_{m=1}^{\nu-1} \frac{\boldsymbol{\Gamma}\left(\widehat{\boldsymbol{q}_{q}} / q-m\right)}{\boldsymbol{\Gamma}\left(\nu+\widehat{\boldsymbol{p}_{p}} / p-m\right)} \\
& \times \frac{Z_{\alpha_{j}}^{m}}{(\nu-1-m)(\nu-2-m) \cdots 2 \cdot 1 \cdot(-1)(-2) \cdots(1-m)} .
\end{aligned}
$$

Since

$$
\begin{aligned}
& \frac{1}{(\nu-1-m)(\nu-2-m) \cdots 2 \cdot 1 \cdot(-1)(-2) \cdots(1-m)} \\
= & \frac{1}{(\nu-1-m) !(-1)^{m-1}(m-1) !} \\
= & \frac{1}{\Gamma(\nu-m)(-1)^{m-1} \Gamma(m)},
\end{aligned}
$$

we have

$$
\begin{aligned}
G_{p, q}^{q, 0}\left(Z_{\alpha_{j}} \begin{array}{c}
\nu+\overline{\boldsymbol{p}} / p \\
\boldsymbol{q} / q
\end{array}\right)= & \sum_{\ell=1}^{q-1} \sum_{n \geq 0} \frac{(-1)^{n}}{n !} \frac{\boldsymbol{\Gamma}\left(\widehat{\boldsymbol{q}_{\ell}} / q-\ell / q-n\right)}{\boldsymbol{\Gamma}(\nu+\overline{\boldsymbol{p}} / p-\ell / q-n)} Z_{\alpha_{j}}^{\ell / q+n} \\
& -\sum_{m=1}^{\nu-1} \frac{\boldsymbol{\Gamma}\left(\widehat{\boldsymbol{q}_{q}} / q-m\right)}{\boldsymbol{\Gamma}(\nu+\overline{\boldsymbol{p}} / p-m)} \times \frac{\left(-Z_{\alpha_{j}}\right)^{m}}{\Gamma(m)}
\end{aligned}
$$


By using the following formula

$$
\Gamma(c-n)=\frac{\Gamma(c)(-1)^{n}}{(1-c)_{n}}, \quad c \in \mathbb{C},
$$

we have

$$
\begin{aligned}
& G_{p, q}^{q, 0}\left(\begin{array}{c|c}
Z_{\alpha_{j}} & \begin{array}{c}
\nu+\overline{\boldsymbol{p}} / p \\
\boldsymbol{q} / q
\end{array}
\end{array}\right) \\
& =Z_{\alpha_{j}}^{\ell / q} \sum_{\ell=1}^{q-1} \frac{\boldsymbol{\Gamma}(\widehat{\boldsymbol{q}} \ell / q-\ell / q)}{\boldsymbol{\Gamma}(\nu+\overline{\boldsymbol{p}} / p-\ell / q)} \sum_{n \geq 0} \frac{(1-\nu-\overline{\boldsymbol{p}} / p+\ell / q)_{n}}{\left(1-\widehat{\boldsymbol{q}_{\ell}} / q+\ell / q\right)_{n}} \frac{\left((-1)^{p-q} Z_{\alpha_{j}}\right)^{n}}{n !} \\
& -\frac{\boldsymbol{\Gamma}\left(\widehat{\boldsymbol{q}_{q}} / q\right)}{\boldsymbol{\Gamma}(\nu+\overline{\boldsymbol{p}} / p)} \sum_{m=1}^{\nu-1} \frac{(1-\nu-\overline{\boldsymbol{p}} / p)_{m}}{\left(1-\widehat{\boldsymbol{q}_{q}} / q\right)_{m}} \frac{\left((-1)^{p-q} Z_{\alpha_{j}}\right)^{m}}{\Gamma(m)} .
\end{aligned}
$$

Finally, by employing the generalized hypergeometric series representation, we obtain the desired result (2.30).

\section{$\S 5 . \quad$ Proof of Corollary 2.5}

If $\arg \alpha_{j}=\theta$ for all $j$, then all the paths of integration for the representation of $u^{d}(t, x)$ in Theorem 2.3 become the same one. Therefore it is enough to prove that the following sum, denoted by $\mathcal{P}(t, \zeta)$, of the polynomial parts $P_{\alpha_{j}}$ disappears

$$
\mathcal{P}(t, \zeta)=\sum_{j=1}^{\mu} \sum_{k=1}^{\ell_{j}} c_{j k} p^{1-k}\left(t \partial_{t}\right)^{k-1} P_{\alpha_{j}}(t, \zeta) .
$$

By substituting the polynomials $P_{\alpha_{j}}$, which are given by (2.32), and carrying out the differentiations, we have

$$
\begin{aligned}
& \mathcal{P}(t, \zeta) \\
= & C_{p, q, \nu} \sum_{m=1}^{\nu-1} \frac{(1-\nu-\overline{\boldsymbol{p}} / p)_{m}}{\left(1-\widehat{\boldsymbol{q}_{q}} / q\right)_{m} \Gamma(m)}\left(\frac{(-1)^{p-q} p^{p} \zeta^{q}}{q^{q}}\right)^{m} t^{-p m} \\
& \times \sum_{j=1}^{\mu} \alpha_{j}^{-m} \sum_{k=1}^{\ell_{j}} c_{j k}(-m)^{k-1} \\
= & C_{p, q, \nu} \sum_{m=1}^{\nu-1} \frac{(1-\nu-\overline{\boldsymbol{p}} / p)_{m}}{\left(1-\widehat{\boldsymbol{q}_{q}} / q\right)_{m} \Gamma(m)}\left(\frac{(-1)^{p-q} p^{p} \zeta^{q}}{q^{q} t^{p}}\right)^{m} A(-m) .
\end{aligned}
$$

Since $A(-m)=0(m=1, \ldots, \nu-1)$, we obtain the desired result. 


\section{§6. Proof of Theorem 2.6}

The proof is done by a similar way to that of Theorem 2.3 except the cases where $(p, q, \nu)=(1,2,1)$ and $(1,3,1)$.

Let $v(s, x)$ be the $(q-p)$ times iterated formal $p$-Borel transform of $\hat{u}(t, x)$ which is given by (2.6)-(2.10) with (2.36), and by the Cauchy integral formula, we have

$$
\begin{aligned}
v(s, x) & =\sum_{n \geq 0} A(n) \varphi^{(q n)}(x) \frac{s^{p n+p \nu-1}}{(p n+p \nu-1) ! \Gamma(1+(p n+p \nu-1) / p)^{q-p}} \\
& =\frac{s^{p \nu-1}}{2 \pi i} \oint_{|\zeta|=r} \frac{\varphi(x+\zeta)}{\zeta} h_{\alpha}(s, \zeta) d \zeta .
\end{aligned}
$$

Here

$$
\begin{aligned}
& =\sum_{n \geq 0} \frac{(\nu)_{n}}{n !} \frac{(q n) !}{(p n+p \nu-1) ! \Gamma(1+(p n+p \nu-1) / p)^{q-p}}\left(\frac{\alpha s^{p}}{\zeta^{q}}\right)^{n} \\
& =\frac{1}{C_{0}}{ }_{q} F_{q-1}\left(\begin{array}{c}
\boldsymbol{q} / q \\
\nu+\widehat{\boldsymbol{p}_{p}} / p, \nu+(p-1) / p, \ldots, \nu+(p-1) / p ; \frac{q^{q}}{p^{p}} \cdot \frac{\alpha s^{p}}{\zeta^{q}}
\end{array}\right),
\end{aligned}
$$

where $C_{0}=\Gamma(p \nu) \Gamma(\nu+(p-1) / p)^{q-p}$. We have the following integral representation

$$
\begin{aligned}
h_{\alpha}(s, \zeta)= & \frac{C_{2}}{2 \pi i C_{0}} \int_{I} \frac{\boldsymbol{\Gamma}(\boldsymbol{q} / q+\tau) \Gamma(-\tau)}{\boldsymbol{\Gamma}\left(\nu+\widehat{\boldsymbol{p}_{p}} / p+\tau\right) \Gamma(\nu+(p-1) / p+\tau)^{q-p}} \\
& \times\left(-\alpha \frac{q^{q} s^{p}}{p^{p} \zeta^{q}}\right)^{\tau} d \tau
\end{aligned}
$$

with a constant

$$
C_{2}=\frac{\boldsymbol{\Gamma}\left(\nu+\widehat{\boldsymbol{p}_{p}} / p\right) \Gamma(\nu+(p-1) / p)^{q-p}}{\boldsymbol{\Gamma}(\boldsymbol{q} / q)} .
$$

In the expression (6.1), by the assumption that $\varphi(x)$ is analytic on $\Omega_{x}$, we can deform the path of integration of $v(s, x)$

$$
v(s, x)=\frac{s^{p \nu-1}}{2 \pi i} \int_{0}^{\infty((d p+\arg \alpha) / q)} \frac{\Phi(x, \zeta)}{\zeta} H_{\alpha}(s, \zeta) d \zeta,
$$

where $\Phi(x, \zeta)$ is given by $(2.26)$ and $H_{\alpha}(s, \zeta)=h_{\alpha}(s, \zeta)-h_{\alpha}\left(s, \zeta \omega_{q}^{-1}\right)$. Therefore the Borel sum $u^{d}(t, x)$ is given by the following iterated Laplace integral

$$
u^{d}(t, x)=\left(\mathcal{L}_{p}^{q-p} v\right)(t, x) .
$$


By exchanging the order of integrations, we have

(6.7) $u^{d}(t, x)=\frac{1}{2 \pi i} \int_{0}^{\infty((p d+\arg \alpha) / q)} \frac{\Phi(x, \zeta)}{\zeta}\left(\mathcal{L}_{p}^{q-p}\left(s^{p \nu-1} H_{\alpha}(s, \zeta)\right)\right)(t, \zeta) d \zeta$.

After the similar calculations in the proof of Theorem 2.3, we have

$$
\begin{aligned}
& \left(\mathcal{L}_{p}^{q-p}\left(s^{p \nu-1} H_{\alpha}(s, \zeta)\right)\right)(t, \zeta) \\
& =\frac{C_{2}}{C_{0}} t^{p \nu-1} \int_{I} \frac{\boldsymbol{\Gamma}\left(\widehat{\boldsymbol{\boldsymbol { q } _ { q }}} / q+\tau\right)}{\boldsymbol{\Gamma}\left(\nu+\widehat{\boldsymbol{p}_{p}} / p+\tau\right)}\left(\alpha \frac{q^{q} t^{p}}{p^{p} \zeta^{q}}\right)^{\tau} d \tau
\end{aligned}
$$

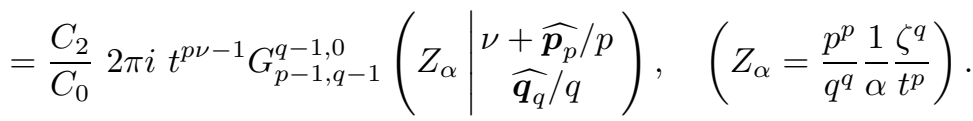

Finally, by using the multiplication formula of the Gamma function, we have

$$
\frac{C_{2}}{C_{0}}=\frac{\boldsymbol{\Gamma}\left(\nu+\widehat{\boldsymbol{p}_{p}} / p\right)}{\Gamma(p \nu) \boldsymbol{\Gamma}(\boldsymbol{q} / q)}=\frac{\boldsymbol{\Gamma}(\boldsymbol{p} / p)}{\Gamma(\nu) \boldsymbol{\Gamma}(\boldsymbol{q} / q) p^{p \nu-1}} .
$$

Thus we obtain the first formula in (2.38), that is, the $G$-function representation of $K_{\alpha}(t, \zeta)$.

The second representation in terms of the generalized hypergeometric function is obtained by taking the residues in the Barnes type integral as in the proof of Proposition 2.4. We omit the detail.

\section{$\S 7 . \quad$ Proof of Corollary 2.7}

Since

$$
\mathcal{K}_{\alpha}(t, \zeta)=\frac{C_{p, q}}{\zeta} G_{p, q}^{q, 0}\left(\begin{array}{c|c}
Z_{\alpha} & \begin{array}{c}
\nu+\overline{\boldsymbol{p}} / p \\
\boldsymbol{q} / q
\end{array}
\end{array}\right)
$$

and

$$
k_{\alpha}(t, \zeta)=\frac{C_{p, q}}{\zeta} G_{p, q}^{q, 0}\left(Z_{\alpha} \begin{array}{l}
\boldsymbol{p} / p \\
\boldsymbol{q} / q
\end{array}\right)
$$

where $Z_{\alpha}=\left(p^{p} / q^{q}\right)(1 / \alpha)\left(\zeta^{q} / t^{p}\right) \in S(0,(q-p) \pi, \infty)$, it is enough to prove the following equality.

$$
\left(\frac{t}{p}\right)^{p \nu-1} G_{p, q}^{q, 0}\left(\begin{array}{c|c}
Z_{\alpha} & \begin{array}{c}
\nu+\overline{\boldsymbol{p}} / p \\
\boldsymbol{q} / q
\end{array}
\end{array}\right)=\left(D_{t}^{-1}\right)^{p \nu-1} G_{p, q}^{q, 0}\left(\begin{array}{l|l}
Z_{\alpha} & \begin{array}{c}
\boldsymbol{p} / p \\
\boldsymbol{q} / q
\end{array}
\end{array}\right),
$$

where $\left(D_{t}\right)^{-1}$ denotes the integration from 0 to $t$. 
By recalling the Barnes type integral expression (2.21), we have

$$
\begin{aligned}
& \left(D_{t}^{-1}\right)^{p \nu-1} G_{p, q}^{q, 0}\left(Z_{\alpha} \begin{array}{l}
\boldsymbol{p} / p \\
\boldsymbol{q} / q
\end{array}\right) \\
& =\left(D_{t}^{-1}\right)^{p \nu-1} \frac{1}{2 \pi i} \int_{I} \frac{\boldsymbol{\Gamma}(\boldsymbol{q} / q+\tau)}{\boldsymbol{\Gamma}(\boldsymbol{p} / p+\tau)}\left(\frac{p^{p} \zeta^{q}}{q^{q} \alpha}\right)^{-\tau} t^{p \tau} d \tau,
\end{aligned}
$$

where the path of integration is taken by $I=\{\tau \in \mathbb{C} ; \operatorname{Re} \tau=\kappa>-1 / q\}$.

We note that the integrand of this integral is absolutely integrable with respect to $t$ with $Z_{\alpha} \in S(0,(q-p) \pi, \infty)$ and $\tau=\kappa+i \eta \in I$. In fact, it follows from the following estimate of the integrand for large $|\tau|$ and $\operatorname{Re} p \tau=p \kappa>$ $-p / q>-1$ : for any fixed $\zeta(\zeta \neq 0)$

$$
\begin{aligned}
& \left|\frac{\boldsymbol{\Gamma}(\boldsymbol{q} / q+\tau)}{\boldsymbol{\Gamma}(\boldsymbol{p} / p+\tau)}\left(\frac{p^{p} \zeta^{q}}{q^{q} \alpha}\right)^{-\tau} t^{p \tau}\right| \\
\leq & \text { Const. }|t|^{p \kappa}|\eta|^{\kappa(q-p)} \exp \left\{-\frac{\pi}{2}(q-p)|\eta|+\eta \arg Z_{\alpha}\right\},
\end{aligned}
$$

which is derived from

$$
\lim _{|y| \rightarrow \infty} \exp \left(\frac{1}{2} \pi|y|\right)|y|^{1 / 2-x}|\Gamma(x+i y)|=(2 \pi)^{1 / 2}, \quad x, y \text { real },
$$

(cf. [Luk, p. 33]).

Therefore by exchanging the order of integrations, we have

$$
\begin{aligned}
& \left(D_{t}^{-1}\right)^{p \nu-1} G_{p, q}^{q, 0}\left(Z_{\alpha} \begin{array}{l}
\boldsymbol{p} / p \\
\boldsymbol{q} / q
\end{array}\right) \\
& =\frac{1}{2 \pi i} \int_{I} \frac{\boldsymbol{\Gamma}(\boldsymbol{q} / q+\tau)}{\boldsymbol{\Gamma}(\boldsymbol{p} / p+\tau)}\left(\frac{p^{p} \zeta^{q}}{q^{q} \alpha}\right)^{-\tau}\left(D_{t}^{-1}\right)^{p \nu-1} t^{p \tau} d \tau .
\end{aligned}
$$

Since

$$
\begin{aligned}
\left(D_{t}^{-1}\right)^{p \nu-1} t^{p \tau} & =\frac{t^{p \tau+p \nu-1}}{(p \tau+1)(p \tau+2) \cdots(p \tau+p \nu-1)} \\
& =\frac{t^{p \tau+p \nu-1}}{p^{p \nu-1}\left(\tau+\widehat{\boldsymbol{p}_{p}} / p\right)_{\nu}(\tau+1)_{\nu-1}} \\
& =\left(\frac{t}{p}\right)^{p \nu-1} \frac{\boldsymbol{\Gamma}(\tau+\boldsymbol{p} / p)}{\boldsymbol{\Gamma}(\tau+\overline{\boldsymbol{p}} / p+\nu)} t^{p \tau}
\end{aligned}
$$


we obtain the desired equality (7.3) as follows:

$$
\begin{aligned}
\left(D_{t}^{-1}\right)^{p \nu-1} G_{p, q}^{q, 0}\left(Z_{\alpha} \mid \begin{array}{c}
\boldsymbol{p} / p \\
\boldsymbol{q} / q
\end{array}\right) & =\left(\frac{t}{p}\right)^{p \nu-1} \frac{1}{2 \pi i} \int_{I} \frac{\boldsymbol{\Gamma}(\boldsymbol{q} / q+\tau)}{\boldsymbol{\Gamma}(\nu+\overline{\boldsymbol{p}} / p+\tau)} Z_{\alpha}^{-\tau} d \tau \\
& =\left(\frac{t}{p}\right)^{p \nu-1} G_{p, q}^{q, 0}\left(\begin{array}{c|c}
\nu+\overline{\boldsymbol{p}} / p \\
Z_{\alpha} \\
\boldsymbol{q} / q
\end{array}\right) .
\end{aligned}
$$

\section{$\S 8 . \quad$ Miscellaneous Remarks}

\section{§8.1. Confluence of roots}

As we have shown, the integral representations of the Borel sum are given in different forms depending on the multiplicity of characteristic roots (see Theorems 2.3 and 2.6). In this subsection we shall show by an example that an integral representation in the case of multiple characteristic roots can be obtained by the confluent procedure from that in the case of distinct characteristic roots.

Let us consider the following Cauchy problem

$$
\left\{\begin{array}{l}
\prod_{j=1}^{3}\left(\partial_{t}^{p}-\alpha_{j} \partial_{x}^{q}\right) u(t, x)=0 \\
\partial_{t}^{k} u(0, x)=0,(k=0,1, \ldots, 3 p-2), \quad \partial_{t}^{3 p-1} u(0, x)=\varphi(x)
\end{array}\right.
$$

where $\varphi(x)$ satisfies the condition for the Borel summability in $d$ direction.

We consider the following three cases:

Case (I): all $\alpha_{j}$ 's are distinct

Case (II): $\alpha_{1}=\alpha_{2}, \alpha_{3} \neq \alpha_{1}$ (confluent procedure $\alpha_{1} \leftarrow \alpha_{2}$ )

Case (III): $\alpha_{1}=\alpha_{2}=\alpha_{3}$ (confluent procedure $\alpha_{1}=\alpha_{2} \leftarrow \alpha_{3}$ )

We only show the confluent procedure from (I) to (II) below, because the confluent procedure from (II) to (III) can be discussed in a similar way.

Case (I). The Cauchy problem (8.1) has a unique formal solution of the form (2.6)-(2.7) with

$$
\begin{aligned}
A(n)= & \frac{\alpha_{1}^{n+2}}{\left(\alpha_{1}-\alpha_{2}\right)\left(\alpha_{1}-\alpha_{3}\right)}+\frac{\alpha_{2}^{n+2}}{\left(\alpha_{2}-\alpha_{1}\right)\left(\alpha_{2}-\alpha_{3}\right)} \\
& +\frac{\alpha_{3}^{n+2}}{\left(\alpha_{3}-\alpha_{1}\right)\left(\alpha_{3}-\alpha_{2}\right)} \\
\stackrel{\text { def }}{=} & c_{1} \alpha_{1}^{n}+c_{2} \alpha_{2}^{n}+c_{3} \alpha_{3}^{n},
\end{aligned}
$$


which is obtained by solving the difference equation (2.8)-(2.9) with $\nu=3$.

By Theorem 2.3, the Borel sum $u^{d}(t, x)$ is given by the following form

$$
\begin{aligned}
u^{d}(t, x) & =\left(\frac{t}{p}\right)^{3 p-1} \sum_{j=1}^{3} c_{j} \int_{0}^{\infty\left(\left(p d+\arg \alpha_{j}\right) / q\right)} \Phi(x, \zeta) \mathcal{K}_{\alpha_{j}}(t, \zeta) d \zeta \\
& \stackrel{\text { def }}{=}\left(\frac{t}{p}\right)^{3 p-1}\left\{c_{1} J_{\alpha_{1}}(t, x)+c_{2} J_{\alpha_{2}}(t, x)+c_{3} J_{\alpha_{3}}(t, x)\right\},
\end{aligned}
$$

where

$$
\mathcal{K}_{\alpha_{j}}(t, \zeta)=\frac{C_{p, q}}{\zeta} G_{p, q}^{q, 0}\left(\begin{array}{c|c}
Z_{\alpha_{j}} & \begin{array}{c}
3+\overline{\boldsymbol{p}} / p \\
\boldsymbol{q} / q
\end{array}
\end{array}\right), \quad Z_{\alpha_{j}}=\frac{p^{p}}{q^{q}} \frac{1}{\alpha_{j}} \frac{\zeta^{q}}{t^{p}}
$$

Case (II). The Cauchy problem (8.1) has a unique formal solution of the form (2.6)-(2.7) with

$$
\begin{aligned}
A(n) & =\frac{\alpha_{1}\left(\alpha_{1}-2 \alpha_{3}\right)}{\left(\alpha_{1}-\alpha_{3}\right)^{2}} \alpha_{1}^{n}+\frac{\alpha_{1}}{\alpha_{1}-\alpha_{3}} n \alpha_{1}^{n}+\frac{\alpha_{3}^{n+2}}{\left(\alpha_{3}-\alpha_{1}\right)^{2}} \\
& \stackrel{\text { def }}{=} c_{11} \alpha_{1}^{n}+c_{12} n \alpha_{1}^{n}+c_{31} \alpha_{3}^{n},
\end{aligned}
$$

which is obtained by solving the difference equation. This is also obtained by taking the confluent procedure $\alpha_{2} \rightarrow \alpha_{1}$ in (8.2), that is,

$$
\begin{aligned}
& c_{1} \alpha_{1}^{n}+c_{2} \alpha_{2}^{n} \longrightarrow c_{11} \alpha_{1}^{n}+c_{12} n \alpha_{1}^{n} \\
& c_{3} \alpha_{3}^{n} \longrightarrow c_{31} \alpha_{3}^{n} .
\end{aligned}
$$

We shall show that the Borel sum is also obtained by taking the confluent procedure from (8.3).

Suppose that $\alpha_{2}$ is so close to $\alpha_{1}$, that is, for a sufficient small $\varepsilon_{1}$, we suppose $\left|\alpha_{1}-\alpha_{2}\right|<\varepsilon_{1}$. Then by the analyticity of the Cauchy data $\varphi(x)$ in $\Omega_{x}$, we can deform the integral path of $J_{\alpha_{2}}(t, x)$ into the integral path of $J_{\alpha_{1}}(t, x)$. Therefore the expression (8.3) is rewritten in the following form

$$
\begin{gathered}
u^{d}(t, x)=\left(\frac{t}{p}\right)^{3 p-1}\left\{\int_{0}^{\infty\left(\left(p d+\arg \alpha_{1}\right) / q\right)} \Phi(x, \zeta) \sum_{j=1}^{2} c_{j} \mathcal{K}_{\alpha_{j}}(t, \zeta) d \zeta\right. \\
\left.+c_{3} \int_{0}^{\infty\left(\left(p d+\arg \alpha_{3}\right) / q\right)} \Phi(x, \zeta) \mathcal{K}_{\alpha_{3}}(t, \zeta) d \zeta\right\} .
\end{gathered}
$$


Since

$$
\begin{aligned}
& \sum_{j=1}^{2} c_{j} \mathcal{K}_{\alpha_{j}}(t, \zeta)=\frac{C_{p, q}}{\zeta} \sum_{j=1}^{2} c_{j} G_{p, q}^{q, 0}\left(\begin{array}{l|c}
Z_{\alpha_{j}} & \begin{array}{c}
3+\overline{\boldsymbol{p}} / p \\
\boldsymbol{q} / q
\end{array}
\end{array}\right) \\
& =\frac{C_{p, q}}{2 \pi i \zeta} \int_{I} \frac{\boldsymbol{\Gamma}(\boldsymbol{q} / q+\tau)}{\boldsymbol{\Gamma}(3+\overline{\boldsymbol{p}} / p+\tau)} Z^{-\tau}\left(\sum_{j=1}^{2} c_{j} \alpha_{j}^{\tau}\right) d \tau
\end{aligned}
$$

with $Z=\left(p^{p} / q^{q}\right)\left(\zeta^{q} / t^{p}\right)$, by taking the confluent procedure $\alpha_{2} \rightarrow \alpha_{1}$, we have

$$
\begin{aligned}
& \sum_{j=1}^{2} c_{j} \mathcal{K}_{\alpha_{j}}(t, \zeta) \\
& \longrightarrow \frac{C_{p, q}}{2 \pi i \zeta} \int_{I} \frac{\boldsymbol{\Gamma}(\boldsymbol{q} / q+\tau)}{\boldsymbol{\Gamma}(3+\overline{\boldsymbol{p}} / p+\tau)} Z^{-\tau}\left(c_{11} \alpha_{1}^{\tau}+c_{12} \tau \alpha_{1}^{\tau}\right) d \tau \\
& =\frac{C_{p, q}}{2 \pi i \zeta}\left\{c_{11} \int_{I} \frac{\boldsymbol{\Gamma}(\boldsymbol{q} / q+\tau)}{\boldsymbol{\Gamma}(3+\overline{\boldsymbol{p}} / p+\tau)} Z_{\alpha_{1}}^{-\tau} d \tau+c_{12} \int_{I} \frac{\boldsymbol{\Gamma}(\boldsymbol{q} / q+\tau)}{\boldsymbol{\Gamma}(3+\overline{\boldsymbol{p}} / p+\tau)} \tau Z_{\alpha_{1}}^{-\tau} d \tau\right\} \\
& \left.=\frac{C_{p, q}}{\zeta}\left\{\begin{array}{l|l}
c_{11} G_{p, q}^{q, 0} & \left(\begin{array}{c|c}
3+\overline{\boldsymbol{p}} / p \\
Z_{\alpha_{1}} / q
\end{array}\right.
\end{array}\right)+c_{12} p^{-1}\left(t \partial_{t}\right) G_{p, q}^{q, 0}\left(\begin{array}{c|c}
Z_{\alpha_{1}} & 3+\overline{\boldsymbol{p}} / p \\
\boldsymbol{q} / q
\end{array}\right)\right\} \\
& =\left(c_{11}+c_{12} p^{-1}\left(t \partial_{t}\right)\right) \mathcal{K}_{\alpha_{1}}(t, \zeta) \text {. }
\end{aligned}
$$

Therefore by noticing $c_{3} \rightarrow c_{31}$, we have

(8.6) $u^{d}(t, x)=\left(\frac{t}{p}\right)^{3 p-1}\left\{\left(c_{11}+c_{12} p^{-1}\left(t \partial_{t}\right)\right) J_{\alpha_{1}}(t, x)+c_{31} J_{\alpha_{3}}(t, x)\right\}$,

which is the one in Theorem 2.3 in the case $\alpha_{1}=\alpha_{2}, \alpha_{3} \neq \alpha_{1}$.

\section{$\S 8.2$. Deformation of paths of integrations of the Borel sum}

As we have shown by the example in subsection 2.12, the Borel sum is the sum of two integrals when $\arg \alpha_{1} \neq \arg \alpha_{2}$. In this subsection, we shall show by the same example $(p=1, q=2)$ that under some additional conditions the Borel sum is given by one integral along a line.

Let us consider the following Cauchy problem

$$
\left\{\begin{array}{l}
\prod_{j=1}^{2}\left(\partial_{t}-\alpha_{j} \partial_{x}^{2}\right) u(t, x)=0 \\
u(0, x)=0, \quad \partial_{t} u(0, x)=\varphi(x)
\end{array}\right.
$$

where we assume $\arg \alpha_{1}<0<\arg \alpha_{2}$ (put $\theta_{j}=\arg \alpha_{j}(j=1,2)$ ), and the Cauchy data $\varphi(x)$ is holomorphic in a neighbourhood of the origin and satisfies 
the conditions for the Borel summability in 0 direction, that is, $\varphi(x)$ can be continued analytically in $\Omega_{x}=\Omega_{x}(1,2,2 ; 0, \varepsilon)=\cup_{j=1}^{2} \cup_{m=0}^{1} S\left(\left(\theta_{j}+2 \pi m\right) / 2, \varepsilon, \infty\right)$ and satisfies

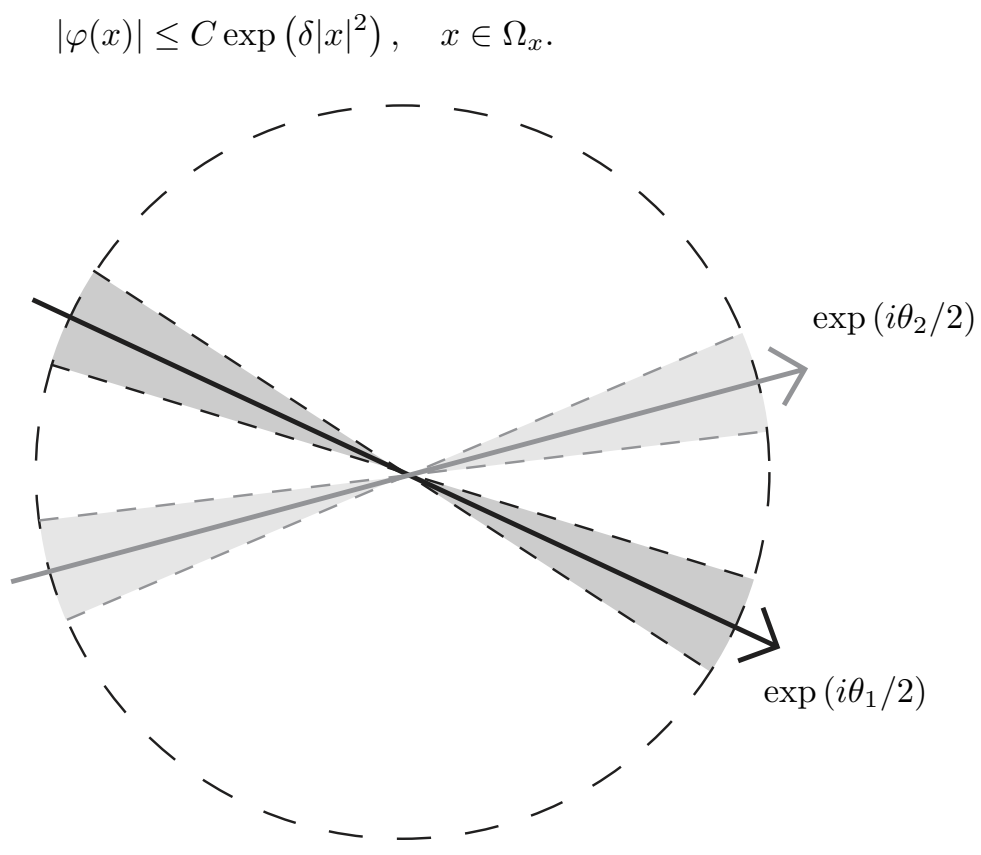

Figure $2 . \Omega_{x}(1,2,2 ; 0, \varepsilon)$

Then the Borel sum $u^{0}(t, x)$ is given by the following function

$$
u^{0}(t, x)=t \sum_{j=1}^{2} c_{j} \int_{e^{i \theta_{j} / 2} \mathbb{R}} \varphi(x+\zeta) \mathcal{K}_{\alpha_{j}}(t, \zeta) d \zeta,
$$

where $(t, x) \in S(0, \alpha, \rho) \times B_{r}$ with $\alpha<\pi$ and sufficiently small $r>0, e^{i \theta_{j} / 2} \mathbb{R}=$ $\left\{\ell e^{i \theta_{j} / 2} ;-\infty<\ell<\infty\right\}, c_{1}=\alpha_{1} /\left(\alpha_{1}-\alpha_{2}\right), c_{2}=\alpha_{2} /\left(\alpha_{2}-\alpha_{1}\right)$ and

$$
\begin{aligned}
(8.10) t \mathcal{K}_{\alpha_{j}}(t, \zeta) & =\frac{t}{\sqrt{\pi} \zeta} G_{1,2}^{2,0}\left(\begin{array}{c|c}
Z_{\alpha_{j}} & 2 \\
1 / 2,1
\end{array}\right), \quad Z_{\alpha_{j}}=\frac{\zeta^{2}}{4 \alpha_{j} t}(\in S(0, \pi, \infty)) \\
& =\sqrt{\frac{t}{\pi \alpha_{j}}}{ }_{1} F_{1}\left(\begin{array}{c}
-1 / 2 \\
1 / 2
\end{array} ;-\frac{\zeta^{2}}{4 \alpha_{j} t}\right)-\frac{\zeta}{2 \alpha_{j}} \quad(\text { by }(2.30)) \\
& =D_{t}^{-1}\left[\frac{1}{\sqrt{4 \alpha_{j} \pi t}} \exp \left(-\frac{\zeta^{2}}{4 \alpha_{j} t}\right)\right] . \quad(\text { by }(2.45))
\end{aligned}
$$


We suppose $0<\theta_{2}-\theta_{1}<\pi$ and we define a multi-sector $\Omega_{x}^{*}$ by

$$
\begin{aligned}
\Omega_{x}^{*} & =\Omega_{x}^{*}\left(\theta_{1}, \theta_{2}\right) \\
& =\bigcup_{m=0}^{1}\left\{S\left(\frac{\theta_{1}+2 m \pi}{2}, \frac{\pi}{2}, \infty\right) \bigcap S\left(\frac{\theta_{2}+2 m \pi}{2}, \frac{\pi}{2}, \infty\right)\right\} \\
& =\bigcup_{m=0}^{1} S\left(\frac{\theta_{1}+\theta_{2}+4 m \pi}{4}, \frac{\pi-\left(\theta_{2}-\theta_{1}\right)}{2}, \infty\right) .
\end{aligned}
$$

We assume the following additional conditions: the Cauchy data $\varphi(x)$ can be continued analytically in $\Omega_{x}^{*}$ (instead of $\Omega_{x}$ ) and satisfies

$$
|\varphi(x)| \leq C \exp \left(\delta|x|^{2}\right), \quad x \in \Omega_{x}^{*} .
$$

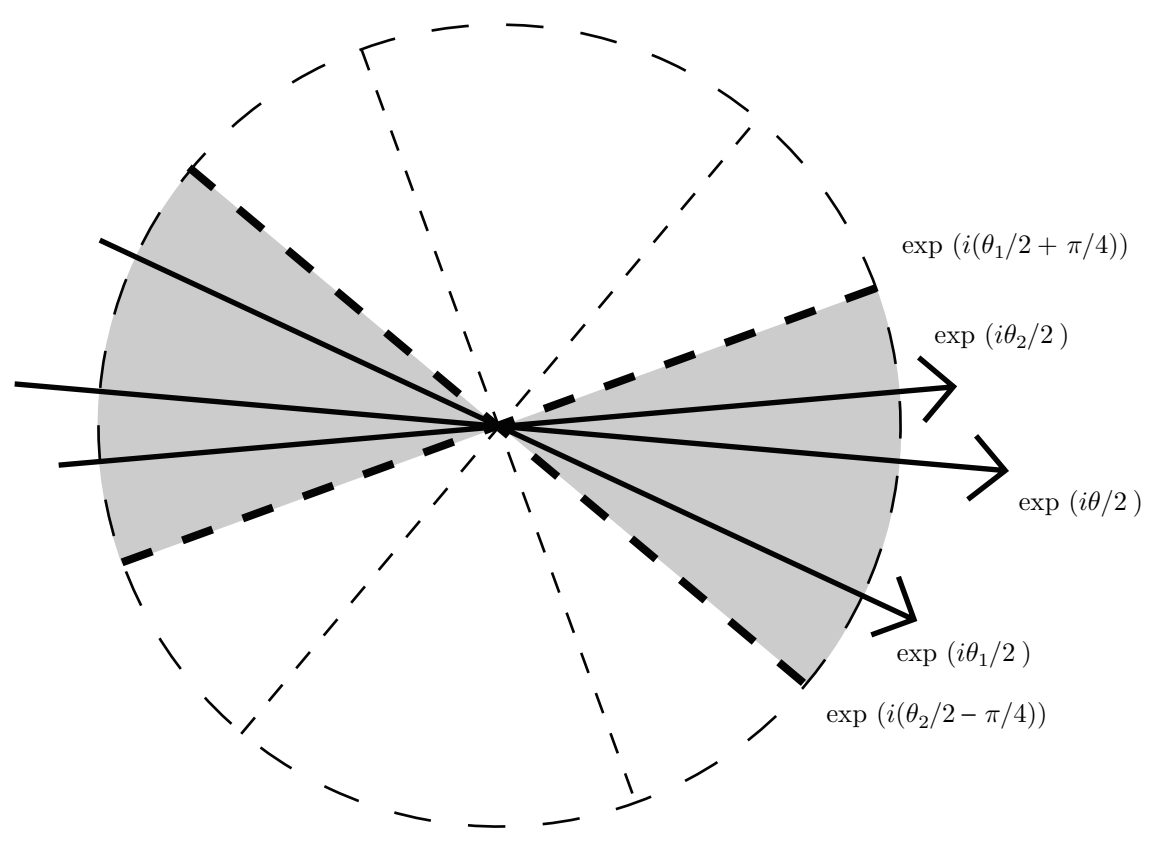

Figure $3 . \Omega_{x}^{*}=\Omega_{x}^{*}\left(\theta_{1}, \theta_{2}\right)$

Under these conditions, when

$$
t \in S\left(\theta-\frac{\theta_{1}+\theta_{2}}{2}, \pi-\left(\theta_{2}-\theta_{1}\right), \rho_{1}\right)
$$


for any fixed $\theta \in\left(\theta_{2}-\pi / 2, \theta_{1}+\pi / 2\right)$ and sufficiently small $\rho_{1}>0$, we can deform all paths of integrations of the Borel sum (8.9) into a line $e^{i \theta / 2} \mathbb{R}$, that is,

$$
\begin{aligned}
u^{0}(t, x) & =\int_{e^{i \theta / 2} \mathbb{R}} \varphi(x+\zeta) \sum_{j=1}^{2} c_{j} t \mathcal{K}_{\alpha_{j}}(t, \zeta) d \zeta \\
& =\int_{e^{i \theta / 2} \mathbb{R}} \varphi(x+\zeta) \sum_{j=1}^{2} c_{j} \sqrt{\frac{t}{\pi \alpha_{j}}}{ }_{1} F_{1}\left(\begin{array}{c}
-1 / 2 \\
1 / 2
\end{array} ;-\frac{\zeta^{2}}{4 \alpha_{j} t}\right) d \zeta
\end{aligned}
$$

where $x \in B_{r^{\prime}}$ for sufficiently small $r^{\prime}(<r)$. The second equality follows from Corollary 2.5, that is, from $c_{1} / \alpha_{1}+c_{2} / \alpha_{2}=0$.

We remark that the expression (8.14) holds for not only $x \in B_{r^{\prime}}$ but also $x \in e^{i \theta / 2} \mathbb{R}$.

In conclusion, this deformation of paths of integrations shows that the region of holomorphy for the Borel sum in $t$-plane is shrunk, that is, the opening angle is actually less than $\pi$, but the region of holomorphy in $x$-plane is spread as mentioned above. Note also that the kernel function in the final integral representation is nothing but the fundamental solution of the Cauchy problem for the parabolic type equation when $\operatorname{Re} \alpha_{j}>0(j=1,2)$.

\section{Appendix. The Proof of Theorem 2.3 in the Case$$
(p, q, \nu)=(1,3,1)
$$

When $(p, q, \nu)=(1,3,1)$, the expression (3.12) is given in the following form.

$$
\begin{aligned}
v(s, x) & =\left(\hat{\mathcal{B}}_{1}^{2} \hat{u}\right)(s, x) \\
& =\frac{1}{2 \pi i} \oint_{|\zeta|=r} \frac{\varphi(x+\zeta)}{\zeta} h_{\alpha}(s, \zeta) d \zeta,
\end{aligned}
$$

where

$$
h_{\alpha}(s, \zeta)={ }_{2} F_{1}\left(\begin{array}{c}
1 / 3,2 / 3 \\
1
\end{array} ; Y_{\alpha}\right), \quad Y_{\alpha}=\frac{3^{3} \alpha s}{\zeta^{3}},
$$

and in $\zeta$-plane $h_{\alpha}(s, \zeta)$ has three singular points at $\zeta=3 \sqrt[3]{\alpha s} \omega_{3}^{m}(m=0,1,2)$ for a fixed $s \neq 0$. We put $3 \sqrt[3]{\alpha s}$ by $\zeta(s)$.

We recall the following Euler's integral representation (cf. [Luk], [IKSY]).

$$
h_{\alpha}(s, \zeta)=\frac{1}{C_{0}} \int_{0}^{1} w^{-1 / 3}(1-w)^{-2 / 3}\left(1-Y_{\alpha} w\right)^{-1 / 3} d w,
$$


where $C_{0}=\Gamma(1 / 3) \Gamma(2 / 3)$ and the branch of the integrand is determined by the following assignment of the arguments: for $w \in(0,1)$ and $\left|Y_{\alpha}\right|<1$,

$$
\arg w=0, \quad \arg (1-w)=0, \quad-\frac{\pi}{2}<\arg \left(1-Y_{\alpha} w\right)<\frac{\pi}{2} .
$$

This integral (A.3) makes sense if $Y_{\alpha} \in \mathbb{C}_{Y_{\alpha}} \backslash\{1\}$ and is a univalent and an analytic function in $\mathbb{C}_{Y_{\alpha}} \backslash[1, \infty)$. Let us determine the branch of the function $\left(1-Y_{\alpha} w\right)^{-1 / 3}$ in $\left|\arg \left(1-Y_{\alpha}\right)\right|=\pi$ with $\left|Y_{\alpha}\right|>1$ (that is, $|\zeta|<3 \sqrt[3]{\alpha|s|}$ in $\zeta$-plane). Then for a fixed $s \neq 0$ with $\arg s=d$, we have

$$
\begin{aligned}
\lim _{\arg \zeta \uparrow(d+\arg \alpha) / 3}\left(1-\frac{3^{3} \alpha s w}{\zeta^{3}}\right)^{-1 / 3} & =\left\{\left(\frac{3^{3} \alpha s w}{\zeta^{3}}-1\right) e^{-\pi i}\right\}^{-1 / 3} \\
\lim _{\arg \zeta \downarrow(d+\arg \alpha) / 3}\left(1-\frac{3^{3} \alpha s w}{\zeta^{3}}\right)^{-1 / 3} & =\left\{\left(\frac{3^{3} \alpha s w}{\zeta^{3}}-1\right) e^{\pi i}\right\}^{-1 / 3}
\end{aligned}
$$

This implies

$$
\begin{aligned}
(\mathrm{A} .7) v(s, x)= & \frac{1}{2 \pi i C_{0}} \int_{0}^{\zeta(s)} \frac{\Phi(x, \zeta)}{\zeta} d \zeta \\
& \times \int_{0}^{1} w^{-1 / 3}(1-w)^{-2 / 3}\left(Y_{\alpha} w-1\right)^{-1 / 3}\left(e^{\pi i / 3}-e^{-\pi i / 3}\right) d w \\
= & \frac{2 i \sin (\pi / 3)}{2 \pi i C_{0}} \int_{0}^{1} w^{-1 / 3}(1-w)^{-2 / 3} d w \\
& \times \int_{0}^{\zeta(s w)} \frac{\Phi(x, \zeta)}{\zeta}\left(Y_{\alpha} w-1\right)^{-1 / 3} d \zeta,
\end{aligned}
$$

where $\Phi(x, \zeta)=\sum_{m=0}^{2} \varphi\left(x+\zeta \omega_{3}^{m}\right), \quad\left(\omega_{3}=e^{2 \pi i / 3}\right)$.

Then the Borel sum $u^{d}(t, x)$ is given by the following iterated Laplace integral

$$
\text { (A.8) } \begin{aligned}
u^{d}(t, x) & =\left(\mathcal{L}_{1}^{2} v\right)(t, x) \\
& =\frac{1}{t} \int_{0}^{\infty(d)} \exp \left(-\frac{s_{1}}{t}\right) d s_{1} \frac{1}{s_{1}} \int_{0}^{\infty(d)} \exp \left(-\frac{s}{s_{1}}\right) v(s, x) d s \\
& =\int_{0}^{\infty(d-\arg t)} e^{-u_{1}} d u_{1} \int_{0}^{\infty(0)} e^{-u_{2}} v\left(u_{1} u_{2} t, x\right) d u_{2},
\end{aligned}
$$

for $t \in S(d, \pi, \rho)$ and $x$ is in a neighbourhood of the origin. 
By exchanging the order of integrations, we have

$$
\begin{aligned}
u^{d}(t, x)= & \frac{\sin (\pi / 3)}{\pi C_{0}} \int_{0}^{\infty((d+\arg \alpha) / 3)} \frac{\Phi(x, \zeta)}{\zeta} d \zeta \\
& \times \int_{0}^{\infty(d-\arg t)} e^{-u_{1}} d u_{1} \int_{0}^{1} w^{-1 / 3}(1-w)^{-2 / 3} d w \\
& \times \int_{1 / A_{\alpha}\left(t, \zeta ; w u_{1}\right)}^{\infty(0)} e^{-u_{2}}\left(A_{\alpha}\left(t, \zeta ; w u_{1} u_{2}\right)-1\right)^{-1 / 3} d u_{2},
\end{aligned}
$$

where

$$
A_{\alpha}(t, \zeta ; X)=\frac{3^{3} \alpha t}{\zeta^{3}} X
$$

By taking a change of variable with respect to $u_{2}$, we obtain

$$
\begin{aligned}
& \int_{1 / A_{\alpha}\left(t, \zeta ; w u_{1}\right)}^{\infty(0)} e^{-u_{2}}\left(A_{\alpha}\left(t, \zeta ; w u_{1} u_{2}\right)-1\right)^{-1 / 3} d u_{2} \\
& =\Gamma\left(\frac{2}{3}\right) A_{\alpha}\left(t, \zeta ; w u_{1}\right)^{-1 / 3} \exp \left(-\frac{1}{A_{\alpha}\left(t, \zeta ; w u_{1}\right)}\right) .
\end{aligned}
$$

Therefore the Borel sum $u^{d}(t, x)$ is given in the following form.

$$
\begin{aligned}
u^{d}(t, x)= & \frac{\sin (\pi / 3) \Gamma(2 / 3)}{\pi C_{0}} \int_{0}^{\infty((d+\arg \alpha) / 3)} \frac{\Phi(x, \zeta)}{\zeta} d \zeta \\
& \times \int_{0}^{\infty(d-\arg t)} e^{-u_{1}} A_{\alpha}\left(t, \zeta ; u_{1}\right)^{-1 / 3} d u_{1} \\
& \times \int_{0}^{1} w^{-2 / 3}(1-w)^{-2 / 3} \exp \left(-\frac{1}{A_{\alpha}\left(t, \zeta ; w u_{1}\right)}\right) d w
\end{aligned}
$$

Here we recall the following formula which follows by taking a change of variable in the integral representation of the Whittaker function.

Formula 1. (cf. [Buc, p. 62]) Suppose $\operatorname{Re} z>0$ and $\operatorname{Re} \beta<1$. Then we have

$$
\int_{0}^{1} t^{-\alpha}(1-t)^{-\beta} e^{-z / t} d t=\Gamma(1-\beta) z^{-\alpha / 2} e^{-z / 2} W_{\mu, m}(z),
$$

where $\mu=\alpha / 2+\beta-1, m=(\alpha-1) / 2$ and the Whittaker function is defined by the following integral

$$
\text { (A.13) } W_{\mu, m}(z)=\frac{e^{-z / 2} z^{\mu}}{\Gamma(1 / 2-\mu+m)} \int_{0}^{\infty} t^{-\mu-1 / 2+m}(1+t / z)^{\mu-1 / 2+m} e^{-t} d t,
$$

with $\operatorname{Re}(\mu-1 / 2-m)<0$ and $|\arg z|<\pi$. 
By using this formula, we obtain

$$
\begin{aligned}
& \int_{0}^{1} w^{-2 / 3}(1-w)^{-2 / 3} \exp \left(-\frac{1}{A_{\alpha}\left(t, \zeta ; w u_{1}\right)}\right) d w \\
& =\Gamma\left(\frac{1}{3}\right) A_{\alpha}\left(t, \zeta ; u_{1}\right)^{1 / 3} \exp \left(-\frac{1}{2 A_{\alpha}\left(t, \zeta ; u_{1}\right)}\right) \\
& \quad \times W_{0,-1 / 6}\left(\frac{1}{A_{\alpha}\left(t, \zeta ; u_{1}\right)}\right) .
\end{aligned}
$$

Hence the Borel sum $u^{d}(t, x)$ is given in the form

$$
\begin{aligned}
u^{d}(t, x)= & \frac{\sin (\pi / 3)}{\pi} \int_{0}^{\infty((d+\arg \alpha) / 3)} \frac{\Phi(x, \zeta)}{\zeta} d \zeta \\
& \times \int_{0}^{\infty(d-\arg t)} e^{-u_{1}} \exp \left(-\frac{1}{2 A_{\alpha}\left(t, \zeta ; u_{1}\right)}\right) \\
& \times W_{0,-1 / 6}\left(\frac{1}{A_{\alpha}\left(t, \zeta ; u_{1}\right)}\right) d u_{1} .
\end{aligned}
$$

Moreover, in order to calculate the integral with respect to $u_{1}$, we use the following formula which gives the relationship between the Whittaker function and the $G$-function.

Formula 2. (cf. [MS, p. 57])

$$
e^{-z / 2} W_{\mu, m}(z)=G_{1,2}^{2,0}\left(z \mid \begin{array}{c}
1-\mu \\
m+1 / 2,-m+1 / 2
\end{array}\right) .
$$

From this formula, we have

$$
\begin{aligned}
& \exp \left(-\frac{1}{2 A_{\alpha}\left(t, \zeta ; u_{1}\right)}\right) W_{0,-1 / 6}\left(\frac{1}{A_{\alpha}\left(t, \zeta ; u_{1}\right)}\right) \\
& =G_{1,2}^{2,0}\left(\frac{1}{A_{\alpha}\left(t, \zeta ; u_{1}\right)} \mid \begin{array}{c}
1 \\
1 / 3,2 / 3
\end{array}\right) .
\end{aligned}
$$


By exchanging the order of integrations between the Laplace integral and Barnes type integral, we obtain

$$
\begin{aligned}
& \int_{0}^{\infty(d-\arg t)} e^{-u_{1}} G_{1,2}^{2,0}\left(\begin{array}{c|c}
\frac{1}{A_{\alpha}\left(t, \zeta ; u_{1}\right)} & 1 / 3,2 / 3
\end{array}\right) d u_{1} \\
& =\int_{0}^{\infty(d-\arg t)} e^{-u_{1}} d u_{1} \\
& \times \frac{1}{2 \pi i} \int_{I} \frac{\Gamma(1 / 3+\tau) \Gamma(2 / 3+\tau)}{\Gamma(1+\tau)} A_{\alpha}\left(t, \zeta ; u_{1}\right)^{\tau} d \tau \\
& =\frac{1}{2 \pi i} \int_{I} \frac{\Gamma(1 / 3+\tau) \Gamma(2 / 3+\tau)}{\Gamma(1+\tau)} A_{\alpha}(t, \zeta ; 1)^{\tau} d \tau \\
& \times \int_{0}^{\infty(d-\arg t)} e^{-u_{1}} u_{1}^{\tau} d u_{1} \\
& =\frac{1}{2 \pi i} \int_{I} \frac{\Gamma(1 / 3+\tau) \Gamma(2 / 3+\tau) \Gamma(1+\tau)}{\Gamma(1+\tau)} A_{\alpha}(t, \zeta ; 1)^{\tau} d \tau \\
& =G_{1,3}^{3,0}\left(\frac{1}{A_{\alpha}(t, \zeta ; 1)} \mid \begin{array}{c}
1 \\
1 / 3,2 / 3,1
\end{array}\right), \quad A_{\alpha}(t, \zeta ; 1)=\frac{3^{3} \alpha t}{\zeta^{3}},
\end{aligned}
$$

where the path of integration $I$ is given by $\{\tau \in \mathbb{C} ; \operatorname{Re} \tau=\kappa, \kappa>-1 / 3\}$.

Hence the Borel sum $u^{d}(t, x)$ is given by the following form.

$$
\begin{aligned}
u^{d}(t, x)= & \frac{\sin (\pi / 3)}{\pi} \int_{0}^{\infty((d+\arg \alpha) / 3)} \\
& \times \frac{\Phi(x, \zeta)}{\zeta} G_{1,3}^{3,0}\left(\frac{\zeta^{3}}{3^{3} \alpha t} \mid \begin{array}{c}
1 \\
1 / 3,2 / 3,1
\end{array}\right) d \zeta .
\end{aligned}
$$

Finally, by noticing

$$
\frac{\sin (\pi / 3)}{\pi}=\frac{1}{\Gamma(1 / 3) \Gamma(2 / 3)},
$$

we obtain the desired formula when $(p, q, \nu)=(1,3,1)$.

\section{Acknowledgement}

The author would like to express his heartfelt thanks to a referee who pointed out a defect in the proof of Theorem 2.3. In order to recover this defect he adds an Appendix where the case of the Airy equation is proved by a different way with other equations. 


\section{References}

[Bal 1] Balser, W., From Divergent Power Series to Analytic Functions, Springer Lecture Notes, No. 1582, 1994.

[Bal 2] Divergent solutions of the heat equations: on the article of Lutz, Miyake and Schäfke, Pacific J. Math., 188 (1999), 53-63.

[BM] Balser, W. and Miyake, M., Summability of formal solutions of certain partial differential equations, Acta. Sci. Math. (Szeged), 65 (1999), 543-551.

[Buc] Buchholz, H., Die Konfluente Hypergeometrische Funktion, Springer-Verlag, 1953.

[Erd] Erdélyi, A., Magnus, W., Oberhettinger, F. and Tricomi, F. G., Higher Transcendental Functions, Vol. I, McGraw-Hill, 1953.

[GT] Gérard, R. and Tahara, H., Singular Nonlinear Partial Differential Equations in the Complex Domain, Vieweg, 1996.

[Ich] Ichinobe, K., The Borel Sum of Divergent Barnes Hypergeometric Series and its Application to a Partial Differential Equation, Publ. RIMS, Kyoto Univ., 37 (2001), 91-117.

[IKSY] Iwasaki, K., Kimura, H., Shimomura, S. and Yoshida, M., From Gauss to Painlevé, A Modern Theory of Special Functions, Aspects Math., Vieweg, Braunschweig, 1991.

[LMS] Lutz, D., Miyake, M. and Schäfke, R., On the Borel summability of divergent solutions of the heat equation, Nagoya Math. J., 154 (1999), 1-29.

[Luk] Luke, Y. L., The Special Functions and Their Approximations, Vol I, Academic Press, 1969.

[Miy 1] Miyake, M., A remark on Cauchy-Kowalevski's theorem, Publ. RIMS, Kyoto Univ., $10(1974 / 75), 243-255$.

[Miy 2] _ Newton polygons and formal Gevrey indices in the Cauchy-Goursat-Fuchs type equations, J. Math. Soc. Japan, 43 (1991), 305-330.

[Miy 3] - Borel summability of divergent solutions of the Cauchy problem to non-Kowalevskian equations, Partial differential equations and their applications (Wuhan, 1999), 225-239, World Sci. Publishing, 1999.

[MS] Mathai, A. M. and Saxena, R. K., Generalized Hypergeometric Functions with Applications in Statistics and Physical Sciences, Springer Lecture Notes, No. 348, 1973.

[Ōuc] Ouchi, S., Genuine solutions and formal solutions with Gevrey type estimates of nonlinear partial differential equation, J. Math. Sci. Univ. Tokyo, 2 (1995), 375-417.

[Shi] Shirai, A., Maillet type theorem for nonlinear partial differential equations and Newton polygons, J. Math. Soc. Japan, 53 (2001), 565-587. 